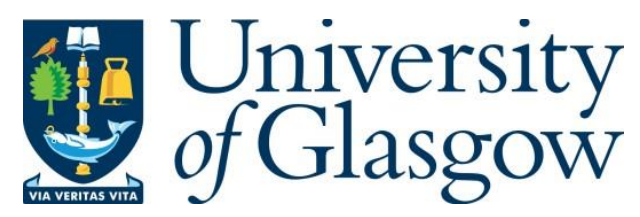

Murray-Smith, D.J. and McGookin, E.W. (2015) A case study involving continuous system methods of inverse simulation for an unmanned aerial vehicle application. Proceedings of the Institution of Mechanical Engineers Part G: Journal of Aerospace Engineering, 229(14), pp. 2700-2717.

There may be differences between this version and the published version. You are advised to consult the publisher's version if you wish to cite from it.

http://eprints.gla.ac.uk/106692/

Deposited on: 26 September 2017

Enlighten - Research publications by members of the University of Glasgow http://eprints.gla.ac.uk 


\title{
A case study involving continuous system methods of inverse simulation for an unmanned aerial vehicle application
}

\author{
D.J. Murray-Smith and E. W. McGookin
}

\begin{abstract}
Inverse simulation allows input time histories to be found that generate specified outputs for nonlinear dynamic models in cases where analytical methods of inversion present difficulties. The two approaches considered involve continuous system simulation principles. One is an approximate differentiation method while the second involves feedback principles. These approaches are compared for a nonlinear six-degrees-offreedom flight-mechanics model of a fixed-wing unmanned aerial vehicle incorporating actuator sub-models with saturation and rate limits. Additional insight is provided through analysis of a linearised version of the vehicle model. It is concluded that both the continuous system simulation methods for finding inverse solutions, for the type of application described in this paper, provide a useful alternative to more conventional iterative methods of inverse simulation based on discrete models. In many cases, including those involving hard nonlinearities in control surface actuator sub-systems, they allow
\end{abstract}


issues of vehicle handling and manoeuvrability to be addressed in a more direct fashion than is possible using conventional simulation methods alone.

\section{Keywords}

Simulation, inverse, nonlinear, model, feedback, differentiation, actuator, aircraft, UAV, manoeuvre.

School of Engineering, University of Glasgow, Glasgow, Scotland, UK.

Corresponding author: D.J. Murray-Smith, School of Engineering, University of Glasgow, Rankine Building, Glasgow G12 8QQ.

E-mail: David.Murray-Smith@Glasgow.ac.uk 


\section{Notation}

A

$A_{\text {lat }}$

$A_{\text {long }}$

$\alpha$

$b$

$b(t)$

B

$B_{\text {lat }}$

$B_{\text {long }}$

$B(s)$

$\beta$

$\bar{c}$ state matrix of linearised state-space model of UAV

state matrix of linearised UAV model for lateral state

variables

state matrix of linearised UAV model for longitudinal state

variables

angle of attack

wing span

input to block diagram used to illustrate feedback method of

inverse simulation

control input matrix of linearised state-space model of UAV

control input matrix of linearised UAV model for lateral state

variables

control input matrix of linearised UAV model for

longitudinal state variables

Laplace transform of $b(t)$

sideslip angle

mean aerodynamic chord 

method of inverse simulation

thrust force derivatives

$C_{L \ldots}$

roll torque derivatives

$C_{M \ldots}$

pitch torque derivatives

$C_{N}$

yaw torque derivatives

$C_{X \ldots}$

drag force derivatives

$C_{Y 1}$

side force derivative

$C_{z 1}, C_{z \alpha}$

lift force derivatives

D

propellor diameter

$\delta$

actuator deflection

$\ddot{o}_{a}$

aileron deflection

$\ddot{o}_{c}$

actuator command input

$\delta_{e}$

elevator deflection

$\ddot{o}_{L}$

actuator input following limiter

$\ddot{o}_{r}$

rudder deflection

$F_{T}$

thrust force 
$g$

$g_{x}^{w}, g_{y}^{w}, g_{z}^{w}$

$G_{a}$

$G_{r}$

$G(s)$

$G_{p A}(s), G_{r A}(s)$

$G_{p R}(s), G_{r R}(s)$

$\bar{h}$

$h(t)$

$h_{1}(s), h_{2}(s)$ component of gravitational vector in $z$ direction in Earth-

fixed frame

$x, y$ and $z$ components of gravitational acceleration referred to

wind frame

gain factor in actuator sub-model associated with actuator

command input

gain factor in actuator sub-model within actuator feedback

loop

linear transfer function

transfer functions from aileron to roll rate and yaw rate in

linearised model of UAV

transfer functions from rudder to roll rate and yaw rate in

linearised model of UAV

parameter used to define manoeuvre amplitude

input variable in block diagram illustrating approximate

differentiation method of inverse simulation

transfer functions within feedback structure for inverse

simulation of linearised lateral-directional model of UAV 


\begin{tabular}{|c|c|}
\hline$K(\mathrm{~s})$ & linear transfer function \\
\hline \multirow[t]{2}{*}{$K_{1}, K_{2}$} & gain factors in feedback structure for inverse simulation of \\
\hline & lateral-directional model of UAV \\
\hline$\theta$ & pitch angle \\
\hline $\boldsymbol{I}$ & aircraft inertia matrix in body-fixed frame (as defined \\
\hline & through moments and products of inertia in Table 3 ) \\
\hline$J$ & thrust advance ratio \\
\hline$L$ & roll torque in body-fixed frame \\
\hline $\bar{m}$ & mass of aircraft \\
\hline \multirow[t]{2}{*}{$m(t)$} & variable within block diagram illustrating approximate \\
\hline & differentiation method of inverse simulation \\
\hline$M$ & pitch torque in body fixed frame \\
\hline$n$ & engine speed \\
\hline$n_{c}$ & desired engine speed \\
\hline$N$ & yaw torque in body fixed frame \\
\hline$p$ & roll rate in body fixed frame \\
\hline$\tilde{p}$ & dimensionless roll rate \\
\hline pdotapp & approximate time derivative of roll rate \\
\hline
\end{tabular}




\begin{tabular}{|c|c|}
\hline$P_{L}, P_{N}, P_{p q}, P_{q r}$ & inertial constants, as defined in Appendix 1 \\
\hline$q$ & pitch rate in body fixed frame \\
\hline$\tilde{q}$ & dimensionless pitch rate \\
\hline$q d o t_{a p p}$ & approximate time derivative of pitch rate \\
\hline$Q_{M}, Q_{p p}, Q_{p r}, Q_{r r}$ & inertial constants, as defined in Appendix 1 \\
\hline$r$ & roll rate in body fixed frame \\
\hline$\tilde{r}$ & dimensionless yaw rate \\
\hline rdotapp & approximate time derivative of yaw rate \\
\hline$R_{L}, R_{N}, R_{p q}, R_{q r}$ & inertial constants, as defined in Appendix 1 \\
\hline$\rho$ & air density \\
\hline$s$ & Laplace variable \\
\hline$S$ & wing surface area \\
\hline$t$ & time \\
\hline$\tau$ & time constant within linear model of actuator \\
\hline$\tau^{\prime}$ & time constant within linear inverse model of actuator \\
\hline$\tau_{n}$ & engine time constant \\
\hline$x$ & vector of state variables \\
\hline$x_{i}$ & $i^{\text {th }}$ state variable within vector $\boldsymbol{x}$ \\
\hline
\end{tabular}




\begin{tabular}{|c|c|}
\hline$x_{i d}$ & desired value of state variable $x_{i}$ \\
\hline$X$ & aerodynamic lateral force (body-fixed frame) \\
\hline$X_{w}$ & aerodynamic lateral force (wind frame) \\
\hline$T$ & time constant of approximate differentiator \\
\hline$T_{m}$ & time period for completion of specified manoeuvre \\
\hline$u$ & inertial velocity component, $\boldsymbol{x}_{b}$ direction \\
\hline$v$ & inertial velocity component, $\boldsymbol{y}_{b}$ direction \\
\hline$v_{\text {des }}(t)$ & required time history of selected angular rate variable \\
\hline$V_{T}$ & airspeed \\
\hline$\phi$ & roll angle \\
\hline$w$ & inertial velocity component, $\boldsymbol{z}_{b}$ direction \\
\hline$w_{1}(t), w_{2}(t)$ & signals representing control surface deflections within \\
\hline & linearised lateral-directional model of UAV \\
\hline$Y$ & aerodynamic drag force (body-fixed frame) \\
\hline$Y_{w}$ & aerodynamic drag force (wind frame) \\
\hline$\psi$ & heading angle \\
\hline$Z$ & aerodynamic lift force (body-fixed frame) \\
\hline$Z_{w}$ & aerodynamic lift force (wind frame) \\
\hline
\end{tabular}




\section{Introduction}

Inverse models can generate time histories of input variables that satisfy given output time history requirements. This has relevance for problems in system dynamics and control, particularly where actuator limits are important. Aircraft and helicopter handling qualities studies and agility investigations are examples where inverse solutions can provide information about manoeuvring problems and about control margins as actuator amplitude or rate limits are approached. ${ }^{1,2}$

In the context of aircraft, the inversion of vehicle dynamics can be used as the design basis of flight control systems and autonomous path planning algorithms. Also, the inverse dynamic solution can be used in the specification process for actuation and sensor systems where appropriate. Although this paper relates to a fixed-wing Unmanned Aerial Vehicle (UAV), the inverse simulation methods described have broad applicability. 


\section{Inverse simulation methods}

Inverse simulation techniques divide into iterative approaches involving discretised models and methods based on continuous system simulation principles that do not involve iteration. Although the second approach is emphasised in this paper, both are discussed since there are links between continuous system simulation approaches and some of the iterative methods involving discretised models.

\section{Iterative methods of inverse simulation}

The iterative technique most widely used was developed by Hess, Gao and Wang and involves repeated solution of a forward simulation model of the vehicle to determine inputs needed to follow a specified manoeuvre. ${ }^{3}$ This is termed an 'integration-based' approach and similar methods were developed independently by Thomson and Bradley and their colleagues. ${ }^{1,4}$ These methods are based mainly on the use of gradient information but search-based optimisation has also been applied successfully. ${ }^{5}$ 
A second iterative method involves a discrete 'differentiation' approach, which was developed by Thomson and his colleagues ${ }^{6,7}$ in the context of helicopter applications and by Kato and Suguira ${ }^{8}$ for fixed-wing aircraft problems. Other iterative techniques were developed for similar applications, including optimisation-based approaches by $\mathrm{Celi}^{9}$ and by Lee and Kim. ${ }^{10}$ A very useful review of iterative methods involving discrete-time models has been provided by Thomson and Bradley. ${ }^{2}$

\section{The continuous system simulation approach to inverse simulation}

Methods of inverse simulation have also evolved that use continuous system simulation principles. Numerical solutions of Differential Algebraic Equations (DAEs) provide one possible approach, ${ }^{11,12}$ but DAE solvers can introduce numerical difficulties and published accounts involve relatively simple low-order models. ${ }^{13}$ Two other approaches to inverse simulation using continuous system simulation principles have been developed. One of these is based on an approximate method of differentiation, while the second involves feedback principles. 


\section{The approximate differentiation method}

Approximate differentiation, using continuous system simulation principles, may be applied over a range of frequencies which must be matched to the frequency range for the specific application. ${ }^{14}$ This is a continuous system equivalent of the discrete 'differentiation' methods mentioned above.

The starting point is the nonlinear model arranged in state-space form, as given in Appendix 1. Equations involving the system inputs are then organised so that the inputs of interest appear on the left hand sides. Derivatives of state variables are then approximated using an integrator block and feedback pathway, as in Figure 1. The firstorder equation defining that system is given by:

$$
\frac{d m}{d t}=\frac{1}{T}(h(t)-m(t))
$$

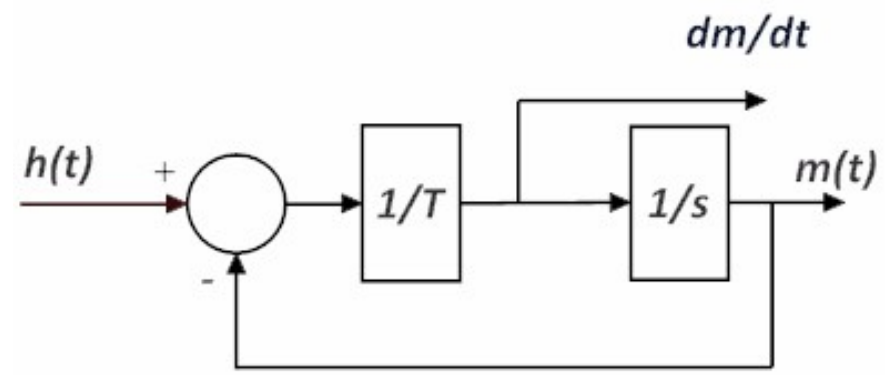

Figure 1. Block diagram of the approximate differentiator using standard continuous 
system simulation elements. The block $1 / s$ represents the operation of integration in terms of the Laplace variable $s$.

The variable $h(t)$ in Figure 1 is first replaced by $x_{i d}(t)$, the desired time history for the variable $x_{i}$. Then, if the time constant $T$ is small in terms of the dynamic response of the model, the variable $m(t)$ in Figure 1 approximates the desired state variable $x_{i d}(t)$. The integrator block input is $\frac{1}{T}\left(x_{i d}(t)-m(t)\right)$ and this is a close approximation to the time derivative of $x_{i d}(t)$. Thus, a derivative of any given state variable, $\dot{x}_{j}$, may be replaced by $\frac{1}{T}\left(x_{j d}(t)-x_{j}(t)\right)$, where $x_{j d}(t)$ is the desired time history. State equations that do not include state variables that are specified for the manoeuvre or input variables that have to be found are not unaltered.

\section{Methods using feedback principles}

The feedback approach to inverse simulation may be explained using a linear model and Laplace transform analysis. The block diagram of Figure 2 involves a single-input singleoutput (SISO) linear model having a transfer function $G(s)$ and a feedback loop having a cascaded block $K(s)$. The transfer function relating the variable $C(s)$ to a reference input $B(\mathrm{~s})$ is given by: 
$\frac{C(s)}{B(s)}=\frac{1}{\frac{1}{K(s)}+G(s)}$

If $1 / K(s)$ is small compared with the magnitude of $G(s)$, over the relevant range of frequencies, the transfer function may be approximated by:

$$
\frac{C(s)}{B(s)} \approx \frac{1}{G(s)}
$$

Thus, if $K(s)$ is large, the transfer function $C(s) / B(s)$ is a close approximation to the inverse model. The same principle applies with nonlinear models and with multi-input multioutput (MIMO) models. Although high-gain feedback often provides acceptable solutions the approach is not limited to proportional control methods.

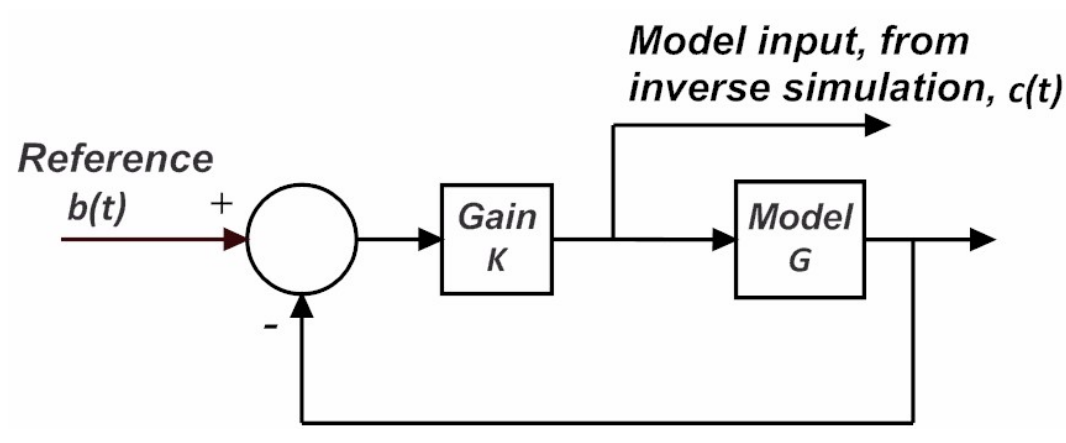


Figure 2. Block diagram for inverse simulation using feedback principles, for a given linear or nonlinear model $G$. For large gain $K$, the variable $c(t)$ closely approximates the model input required to produce an output that matches the reference time history $b(t)$.

The feedback approach originates from methods use in dividers and inverse function generators in electronic analogue computers. For inverse modelling, some early applications of feedback can be found in work carried out at the DLR aerospace research institute in Germany. ${ }^{15}$ - 17 A broadly similar approach, termed 'inverse dynamics compensation via simulation of feedback control systems' (IDCS) was developed by Tagawa and Fukui in Japan. ${ }^{18}$ Further developments in terms of these feedback-based methods and their application to practical problems have been reported recently. ${ }^{19-23}$

One problem with the feedback method is hard limiting which produces limit cycles which are not a property of the inverse model and arise from the discontinuities and the feedback structure. ${ }^{23}$ Rate limits are a particular problem in terms of limit cycle oscillations and this may be understood from the fact that the describing function for this nonlinearity has both imaginary and real components and net phase lags of the model output in excess of -180 deg (relative to the input) are potentially troublesome. ${ }^{24}$ High-order models are also more likely to present difficulties than low order models in terms of limit cycles and instabilities introduced by feedback. 
Although limit cycling and instability may occur with the feedback approach, it should be noted that feedback design for inverse simulation is generally easier than the design of feedback control systems. In control applications designers must allow for disturbance rejection, measurement noise and model uncertainty, in addition to issues of stability and dynamic response. Thus, although high gain solutions may not lead to robust control systems, they often provide satisfactory outcomes for inverse simulation applications.$^{21-}$ 23

\section{An Application to an Unmanned Aerial Vehicle (UAV) model.}

The application concerns a nonlinear model of a small UAV of mass $28 \mathrm{~kg}$ and wing-span $3.1 \mathrm{~m} .{ }^{25,26}$ Typical flight conditions involve a forward speed of $30 \mathrm{~m} / \mathrm{s}$. The reference frames and associated dynamic variables are illustrated in Figure 3. 


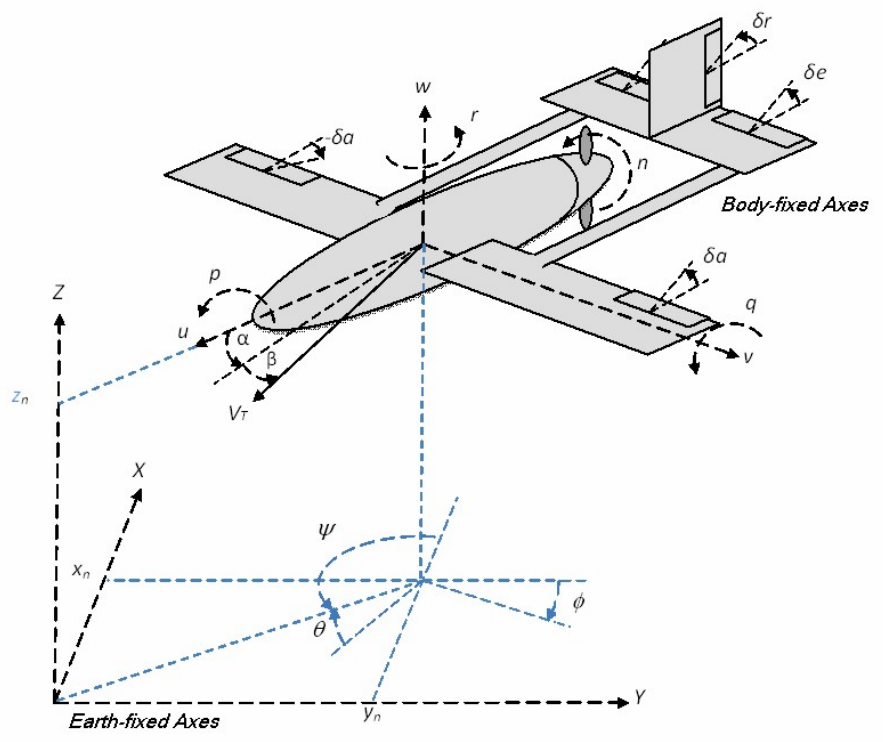

Figure 3. UAV reference frames and dynamic variables

Details of the state variables of the model are given in Table 1 and inputs are defined inTable 2. 
Table 1. State variables of the model.

\begin{tabular}{|c|c|c|c|c|c|}
\hline Variable & Description & Units & Variable & Description & Units \\
\hline$u$ & Longitudinal & $\mathrm{m} / \mathrm{s}$ & $x_{n}$ & x-coordinate position & $\mathrm{m}$ \\
\hline$v$ & Lelocity & & & & \\
\hline$w$ & Vertical velocity & $\mathrm{m} / \mathrm{s}$ & $z_{n}$ & Altitude & $\mathrm{m}$ \\
\hline$p$ & Roll rate & $\mathrm{rad} / \mathrm{s}$ & $\phi$ & Roll angle & radians \\
\hline$q$ & Pitch rate & $\mathrm{rad} / \mathrm{s}$ & $\theta$ & Pitch angle & radians \\
\hline$r$ & Yaw rate & $\mathrm{rad} / \mathrm{s}$ & $\psi$ & Yaw angle & radians \\
\hline$\alpha$ & Angle of attack & $\mathrm{radians}$ & & & \\
\hline$\beta$ & Sideslip angle & $\mathrm{radians}$ & & & \\
\hline$V_{T}$ & Airspeed & $\mathrm{m} / \mathrm{s}$ & & & \\
\hline
\end{tabular}


Table 2. Input variables of the model.

\begin{tabular}{|c|l|l|}
\hline Input & Description & Units \\
\hline$\delta_{a}$ & Aileron deflection angle & radians \\
\hline$\delta_{e}$ & Elevator deflection angle & radians \\
\hline$\delta_{r}$ & Rudder deflection angle & radians \\
\hline$n$ & Engine speed & $\mathrm{rev} / \mathrm{s}$ \\
\hline
\end{tabular}

A nonlinear six degrees-of-freedom description, relating state variables to control surface deflections and engine speed, is provided in Appendix 1 and typical parameter values are given in Table $3 .^{25}$

The model of Appendix 1 does not include actuator sub-models. Any actuator will have well-defined amplitude and rate limits which can limit performance. ${ }^{24}$ Generalised actuator sub-models have been proposed previously, involving simplified block diagram representations of the form shown in Figure $4 .^{27}$ 


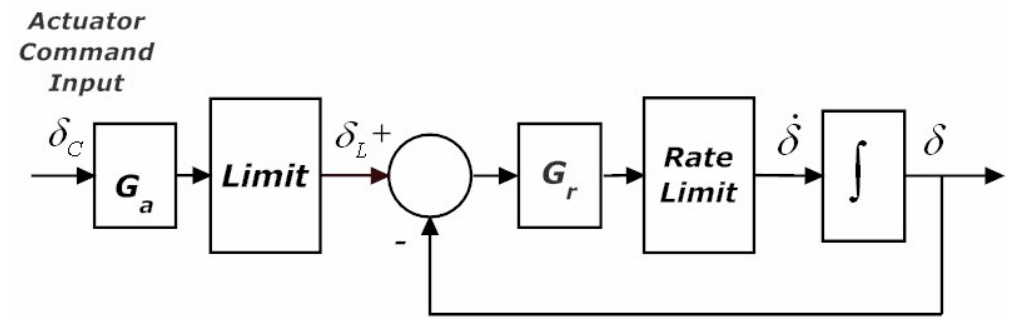

Figure 4. A simplified block diagram of an actuator sub-model with amplitude and rate limiting. The signal $\delta_{c}$ is the actuator command input prior to any limiting. The signal $\delta_{L}$ is the actuator input following the saturation limiter and $\delta$ represents the control surface position.

The value of the gain constant $G_{r}$ is chosen to be 10 for this application, which corresponds to an actuator time constant in the linear mode of operation of $0.1 \mathrm{~s}$. The value for the gain factor $G_{a}$ is unity. The saturation limiter has a simple form and when its input $G_{a} \delta_{C}$ lies in the range between the upper and lower saturation limits $\left(\delta_{\mathrm{cU}}\right.$ and $\left.\delta_{\mathrm{cL}}\right)$ it behaves as a linear element with unit gain,. However, if $G_{a} \delta_{C} \geq \delta_{\mathrm{cU}}$ the output value is limited at $\delta_{\mathrm{cU}}$ and, correspondingly, when $G_{a} \delta_{C} \leq \delta_{\mathrm{cL}}$ the output is limited at $\delta_{\mathrm{cL}}$. This behaviour is usually symmetrical for positive and negative inputs with $\delta_{\mathrm{cU}}=-\delta_{\mathrm{cL}}$. The rate limit block has an identical form, having unity gain when the rate of change of actuator ouput position $(\dot{\delta}(t))$ has a value that lies within the specified upper and lower rate limits. The block 
diagram can be modified for actuators with second-order characteristics within the linear range. In that case the block labelled $G_{\mathrm{r}}$ within the feedback loop of Figure 4 would have first-order lag characteristics.

\section{Inverse simulation results}

As shown in Table 2, the model has four inputs, involving engine speed, elevator angle, aileron angles and rudder angle. Note that the left and right aileron control surfaces operate in a differential sense, whereas the elevator surfaces move in the same direction. In this application the engine speed is kept constant and only the three control surface inputs are considered.

Results are divided between cases involving linear operation of the control surface actuators and situations in which the control surface actuators reach one or both limits.

The chosen flight conditions involve an initial forward velocity component in the body axis system of $32.57 \mathrm{~m} / \mathrm{s}$ and a vertical velocity component of $2.57 \mathrm{~m} / \mathrm{s}$, corresponding to an angle of attack of $0.07 \mathrm{deg}$. and an initial elevator deflection of $-1.438 \mathrm{deg}$. 


\section{Situations involving actuators within the linear operating range}

In this case the actuators for the ailerons, elevator and rudder are all modelled as linear systems with transfer function:

$$
\frac{\delta(s)}{\delta_{L}(s)}=\frac{1}{1+s \tau}
$$

Equation (4) is equivalent to the model of Figure 4 with $\tau=\frac{1}{G_{r}}$ and $G_{a}=1$ for situations where each actuator sub-system operates linearly.

Manoeuvres may be specified in terms of demanded time histories of the roll rate, pitch rate and yaw rate variables. In this application these variables are specified as being zero or having a bell-shaped time history which is described by a polynomial:

$$
v_{\text {des }}(t)=\left[30\left(\frac{t}{T_{m}}\right)^{4}-60\left(\frac{t}{T_{m}}\right)^{3}+30\left(\frac{t}{T_{m}}\right)^{2}\right] \frac{\bar{h}}{T_{m}}
$$

where $T_{m}$ is the time taken to complete the manoeuvre and $v_{\text {des }}(t)$ is the required time history of the selected angular rate variable. Polynomials of this form give smooth transitions at the entry and exit points of the manoeuvre. ${ }^{2}$ For the polynomial of equation 
(5) the maximum magnitude of the desired change occurs at time $t=T_{m} / 2$ with value $\frac{\bar{h}}{T_{m}} \times \frac{15}{8}$. For example, for $T_{m}=3$ and $\bar{h}=15$ the maximum angular velocity due to the applied input is $9.375 \mathrm{deg} / \mathrm{s}$. This could be used to describe a pop-up manoeuvre or a transient change of heading.

\section{Results for the linear case using the approximate differentiation approach}

Application of the approximate differentiation approach to equations (22) - (27) of Appendix 1, involves a model in which the three state equations involving time derivatives of angular velocities in the body-fixed frame (equations (25) - (27)) and the aerodynamic torques $L, M$ and $N$ are replaced. Three new equations ((6) - (8) below) implement the approximate differentiation of the roll, pitch and yaw rate variables.

$$
\begin{aligned}
& \dot{p}=\frac{1}{T}\left(p_{\text {des }}-p\right) \\
& \dot{q}=\frac{1}{T}\left(q_{\text {des }}-q\right) \\
& \dot{r}=\frac{1}{T}\left(r_{\text {des }}-r\right)
\end{aligned}
$$

where $T$ is the time constant of the approximate differentiator. From Appendix 1, the roll, pitch and yaw torques in the body-fixed frame, $L, M$ and $N$ respectively, may then be found from the equations: 


$$
\begin{gathered}
L=\frac{1}{P_{L}}\left(p d o t_{a p p}-P_{p q} p q-P_{q r} q r-P_{N} N\right) \\
M=\frac{1}{Q_{M}}\left(q d o t_{a p p}-Q_{p p} p^{2}-Q_{p r} p r-Q_{r r} r^{2}\right) \\
N=\frac{P_{L}}{\left(R_{N} P_{L}-R_{L} P_{N}\right)}\left(\operatorname{rdot}_{a p p}-\frac{R_{L}}{P_{L}} p d o t_{a p p}+\left(\frac{R_{L}}{P_{L}} P_{q r}-R_{p q}\right) p q-\left(R_{q r}-\frac{R_{L}}{P_{L}} P_{q r}\right) q r\right)
\end{gathered}
$$

Here the variables $p d o t_{a p p}, q d o t_{a p p}$ and $r d o t_{a p p}$ are the approximate derivatives, as calculated from the expressions on the right hand sides of equations $(6)-(8)$, and other variables and parameters are as given in Appendix 1. From equations (9) - (11) the control surface deflections are found using equation (40), giving the three algebraic equations needed to complete the set of equations for inverse simulation, together with the remaining state equations of Appendix 1 ( equations (22) - (24) and (28) - (37)).

Figure 5 shows results for the case involving zero values of desired roll rate and pitch rate variables $\left(p_{d e s}\right.$ and $\left.q_{d e s}\right)$ and a desired yaw rate $\left(r_{d e s}\right)$ having the form of equation (5) with parameter values of $\bar{h}$ and $T_{m}$ of 15 and 3 respectively, giving a maximum demanded yaw rate of $9.375 \mathrm{deg} / \mathrm{s}$ at time $t=1.5 \mathrm{~s}$. The time constants, $T$, used for differentiation were $0.001 \mathrm{~s}$. Figure 5 also shows the pitch, roll and yaw rate variables for this manoeuvre 
together with the desired time histories. The yaw rate response is slightly delayed compared with the corresponding desired response. This is due to the actuator time constant which has a value of $0.1 \mathrm{~s}$, corresponding to a value of 10 for the $G_{r}$ parameter value in the block diagram of Figure 4.
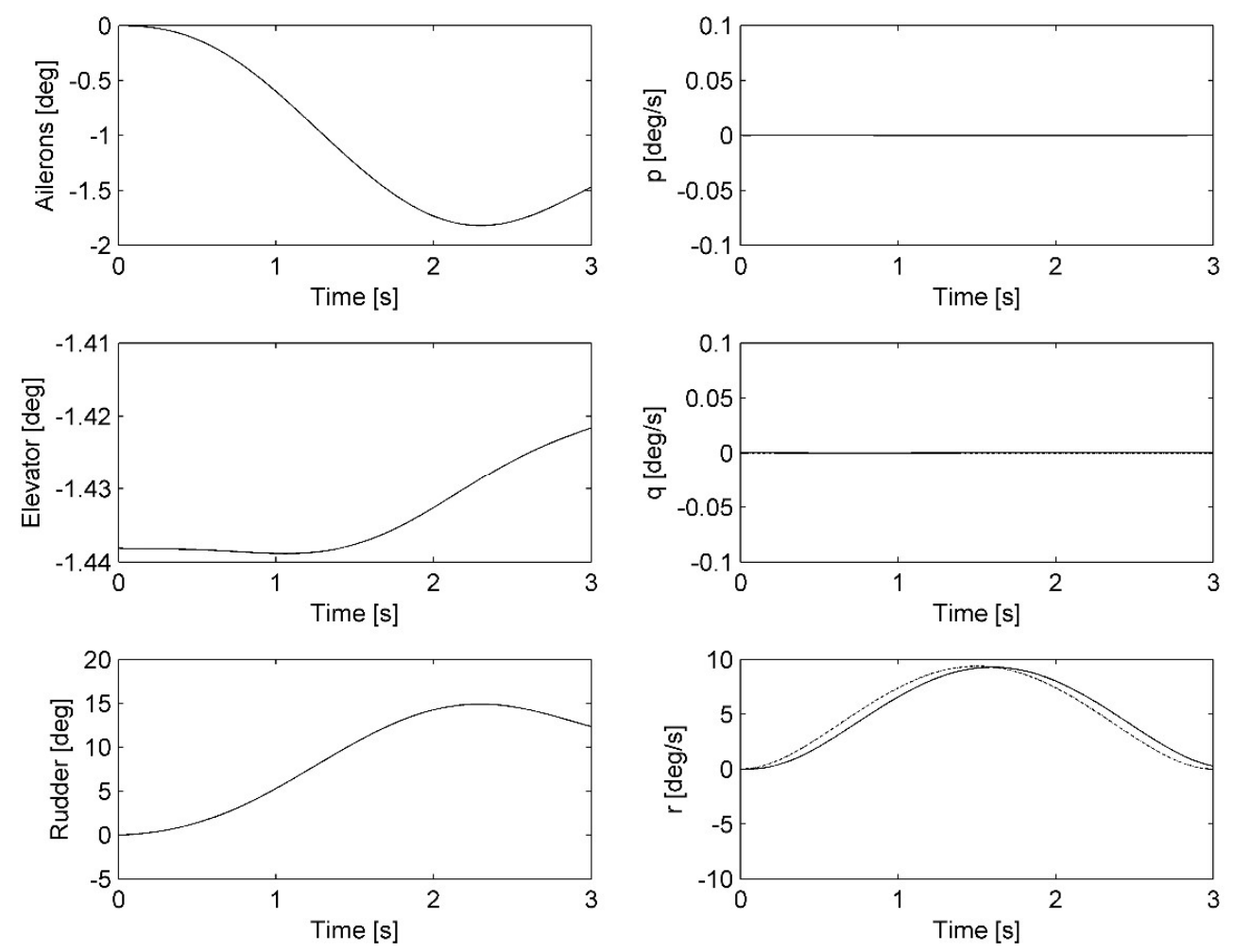

Figure 5. Angular positions (deg) of ailerons, elevator and rudder for the demanded manoeuvre involving a maximum yaw rate of $9.375 \mathrm{deg} / \mathrm{s}$, found from inverse simulation 
using approximate differentiation. Also shown are the roll, pitch and yaw rate variables resulting from the application of these inputs. The desired time histories for these variables are shown by dashed lines.

Roll, pitch and yaw angles found from the inverse simulation are shown in Figure 6 together with the forward, lateral and vertical velocities in the body-fixed frame. 

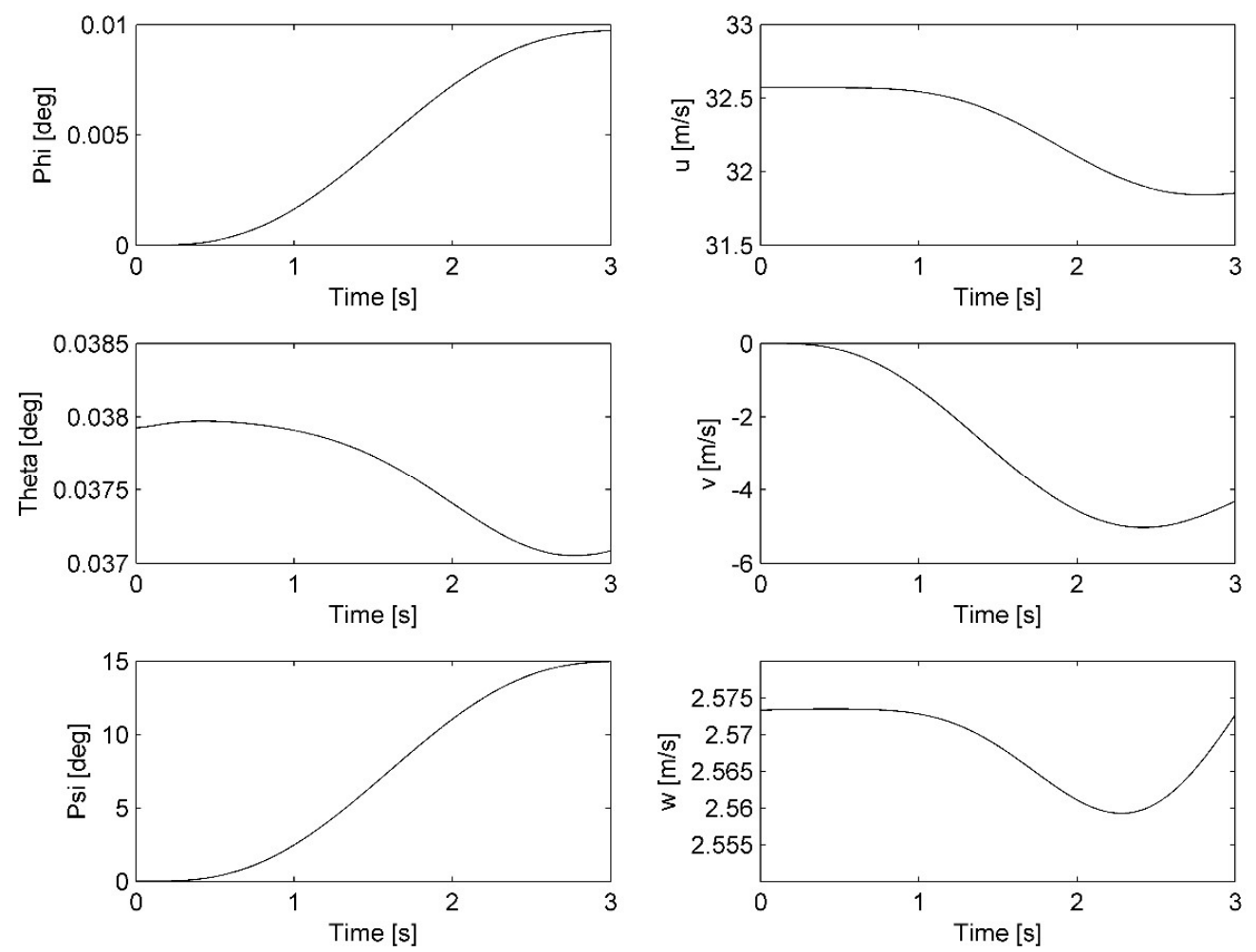

Figure 6. Roll, pitch and yaw angles for the control inputs found from inverse simulation results of Figure 5, together with the forward, lateral and vertical velocities of the vehicle in the body-fixed frame of reference.

These results can be understood from analysis of the UAV model of Appendix 1. Equations defining the angular accelerations, as represented in equations (25)-(27), 
involve products of the angular rates $p$ and $q, p$ and $r$ and $q$ and $r$. These products disappear if we constrain the angular rates $p$ and $q$ to be zero, giving:

$$
\begin{gathered}
\frac{I_{z z}}{I_{x x}} L+\frac{I_{x z}}{I_{x x}} N=0 \\
\frac{1}{I_{y y}} M+\frac{I_{x z}}{I_{y y}} r^{2}=0 \\
\frac{I_{x z}}{I_{r r}} L+\frac{I_{x x}}{I_{r r}} N=\dot{r}
\end{gathered}
$$

where $I_{r r}=I_{x x} I_{z z}-I_{x z}^{2}$. The $L, M$ and $N$ moments depend on the aileron, elevator and rudder deflections respectively and on other terms which are known. From equation (12), it follows that:

$$
L=-\frac{I_{x z}}{I_{z z}} N
$$

and thus, from equation (14),

$$
N=I_{z z} \dot{r}
$$

Thus, from equation (16), the rudder deflection follows the form of the demanded heading rate change and from equation (15) the pattern of aileron deflection can be expected to be similar in shape to the pattern of rudder deflection, but with the opposite sign, This can be observed in Figure 5 where the rudder deflection has the same general shape as the 
demanded heading rate change (but shifted slightly in terms of time) and the aileron deflection is similar to the rudder deflection, but opposite in sign.

Actuator inputs for these required control surface movements are found by inverting the linear actuator sub-model of equation (4) to give an approximate inverse transfer function:

$$
\frac{\delta_{L}(s)}{\delta(s)}=\frac{1+s \tau}{1+s \tau^{\prime}}
$$

The pole at $s=-\frac{1}{\tau^{\prime}}$ in the s-plane (which must be far to the left of the pole at $s=-\frac{1}{\tau}$ in equation (4)) is necessary to give a realisable transfer function. The value of $\tau^{\prime}$ is chosen as $0.001 \mathrm{~s}$ since that is considered small enough compared with the time constant $\tau$ which is $0.1 \mathrm{~s}$ for all three actuators.

\section{Results for the linear case using the feedback approach}

The inverse simulation cases considered all involve three feedback loops, each with an actuator sub-model. The first loop involves feedback of the pitch rate variable, comparison with a desired pitch rate and simple proportional feedback control to the input of the actuator sub-model for the elevator. The second and third loops are similar to this, 
but involve the roll-rate variable and the sub-model of the aileron actuator in the second case and heading rate together with the rudder actuator sub-model in the third.

Gain factors in all three loops have been chosen to be $10^{4}$, which is a compromise between accuracy and computational efficiency. These gain factor values are also used for all the other cases considered in this paper using the feedback approach.
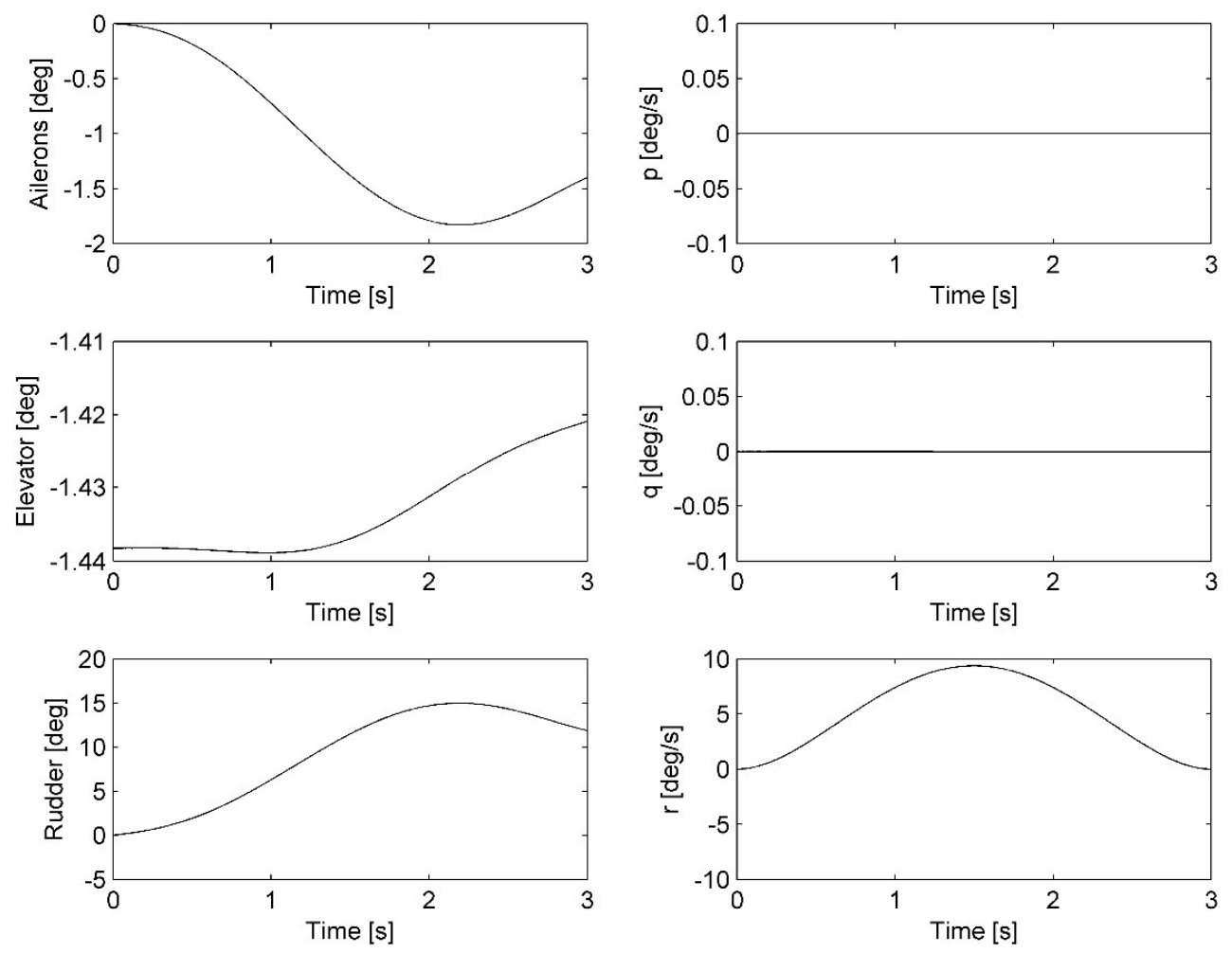
Figure 7. Angular positions (deg) of ailerons, elevator and rudder for the demanded manoeuvre involving a maximum yaw rate of $9.375 \mathrm{deg} / \mathrm{s}$., as found by inverse simulation using the feedback approach. Also shown are the roll, pitch and yaw rates resulting from these inputs. The desired time histories are shown by dashed lines but, in this case, these coincide with the plots for the angular rate variables.

The results of Figure 7 are almost indistinguishable, graphically, from those found by approximate differentiation and shown in Figure 5, except that they do not show any effects of the actuator time constants observed with the approximate differentiation method. Other state variable plots, such as those for the attitude angles and the velocity components, are very similar to the approximate differentiation results of Figure 6. Similar findings are obtained for other forms of manoeuvre, involving demanded roll rate or pitch rate time histories and for more complex combinations of demanded variables, but without limiting.

\section{Situations involving actuator saturation and rate limiting effects}

Actuator limits for normal operation were chosen to be $\pm 20 \mathrm{deg}$. for control surface deflections and $\pm 30 \mathrm{deg} / \mathrm{s}$ for rate limits. The actuator sub-model time constant was $0.1 \mathrm{~s}$, 
as before. Increasing the maximum demanded heading rate gives a manoeuvre having the same shape as the manoeuvre considered previously until the rudder deflection reaches the limit of $20 \mathrm{deg}$. Similarly, reducing the time period for the manoeuvre leads, eventually, to rudder rate limiting. Then any further increase in demanded heading rate produces no further change of rudder position. Similar limiting effects are found with demanded aileron and elevator inputs in terms of roll rates or pitch rates respectively.

\section{Inverse simulation results with actuator limiting by the approximate differentiation approach.}

Although actuator inputs can be found from the required control surface deflections in the case of a linear actuator model, this form of inversion process for the actuator sub-model is inapplicable with limiting. A two-stage approach, which first involves finding the inputs that would have to be applied to the actuators for linear operation, is necessary. These inputs are then applied, in the second stage, to the UAV model with the nonlinear actuator sub-model included to find how amplitude and rate limits affect the control surface deflections and other variables. This procedure allows the time of occurrence of limiting to be investigated and the ways in which the limits affect the other state variables of the 
UAV model can also be observed. Figure 8 is a block diagram that illustrates the approach used.

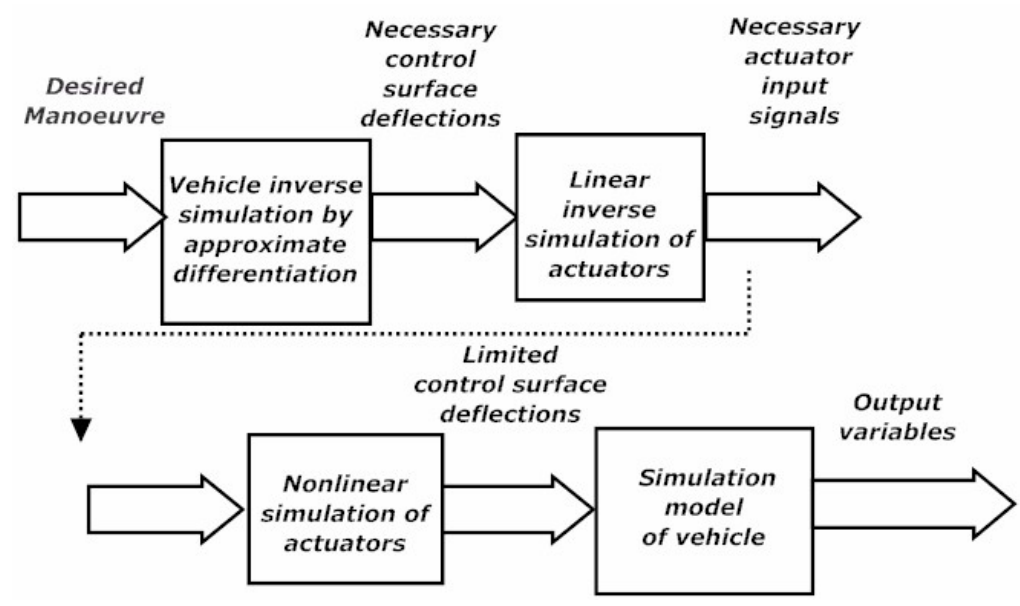

Figure 8. Block diagram showing the two-stage procedure used for inverse simulation by the approximate differentiation method, with actuator nonlinearities included only in the second stage involving forward simulation.

Results obtained using this two-stage approach are shown in Figure 9 for the type of manoeuvre considered previously, but with the amplitude of demanded yaw rate increased so that the rudder actuator reaches its amplitude and rate limits during the manoeuvre. 

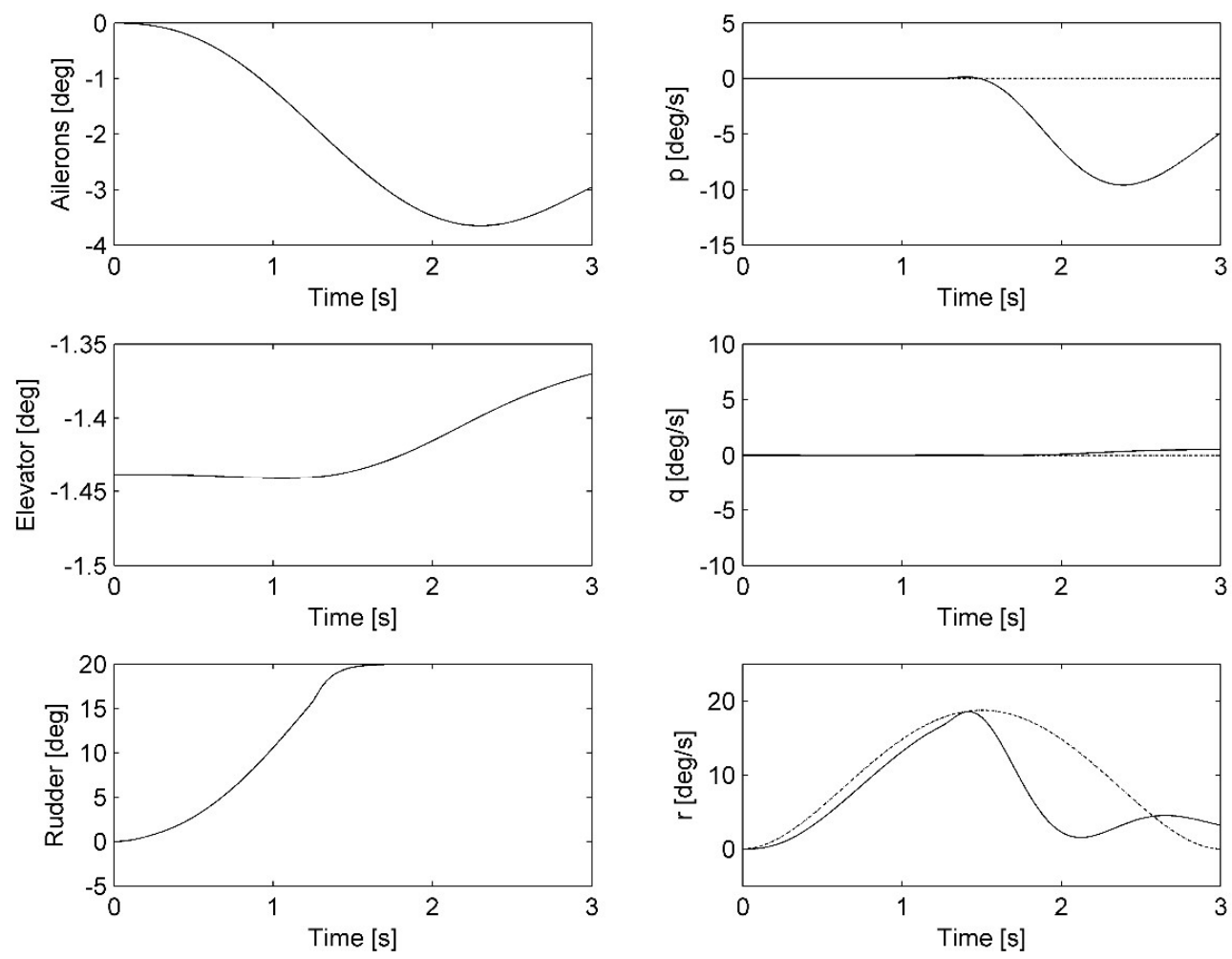

Figure 9. Control surface deflections and attitude angular velocities found by the approximate differentiation method of inverse simulation for case of a manoeuvre involving a maximum yaw rate of $18.75 \mathrm{deg} / \mathrm{s}$, amplitude limits of $\pm 20 \mathrm{deg}$ and rate limits of $\pm 30 \mathrm{deg} / \mathrm{s}$. The desired angular velocities are shown by the dashed lines. 
In Figure 9 the effect of the saturation limit of 20 deg. on the rudder deflection is clear. The form of the aileron and elevator deflections resemble those of Figures 5 and 7 but the aileron deflection is greater. This is expected as the manoeuvre in this case is much larger. In terms of the angular rates, it can be seen in Figure 9 that both the roll rate $(p)$ and pitch rate $(q)$ remain close to the desired values of zero until the rudder reaches saturation at about $t=1.5 \mathrm{~s}$. Then, while the rudder remains in a saturated condition, the values of $p$ and $q$ start to diverge from the required values of zero. The yaw rate time history also diverges significantly from that desired, due to the rudder-actuator nonlinearities, but reaches a maximum of about $18 \mathrm{deg} / \mathrm{s}$ which is close to the demanded maximum of 18.75 $\operatorname{deg} / \mathrm{s}$.

Records for the attitude angles $\phi, \theta$ and $\psi$ when the control surface inputs found from inverse simulation are applied to the vehicle model show, as would be expected, larger changes from the trimmed state than in the case considered previously in which actuator limits were not reached. The effects of rate limiting can be seen more clearly by considering a larger manoeuvre with the rudder actuator rate limit changed from $\pm 30 \mathrm{deg} / \mathrm{s}$ to $\pm 10 \mathrm{deg} / \mathrm{s}$. Figure 10 shows the control surface deflections and resulting attitude rates for these limits for a case involving a demanded manoeuvre with a maximum yaw rate of $56.25 \mathrm{deg} . / \mathrm{s}$. The limiting of the rudder rate is very obvious in this case and divergence 
of the attitude rates of the vehicle from the desired values may also be seen. Due to the rate limit the maximum yaw rate achieved is about $9 \mathrm{deg} / \mathrm{s}$ which is about one sixth of the demanded value.
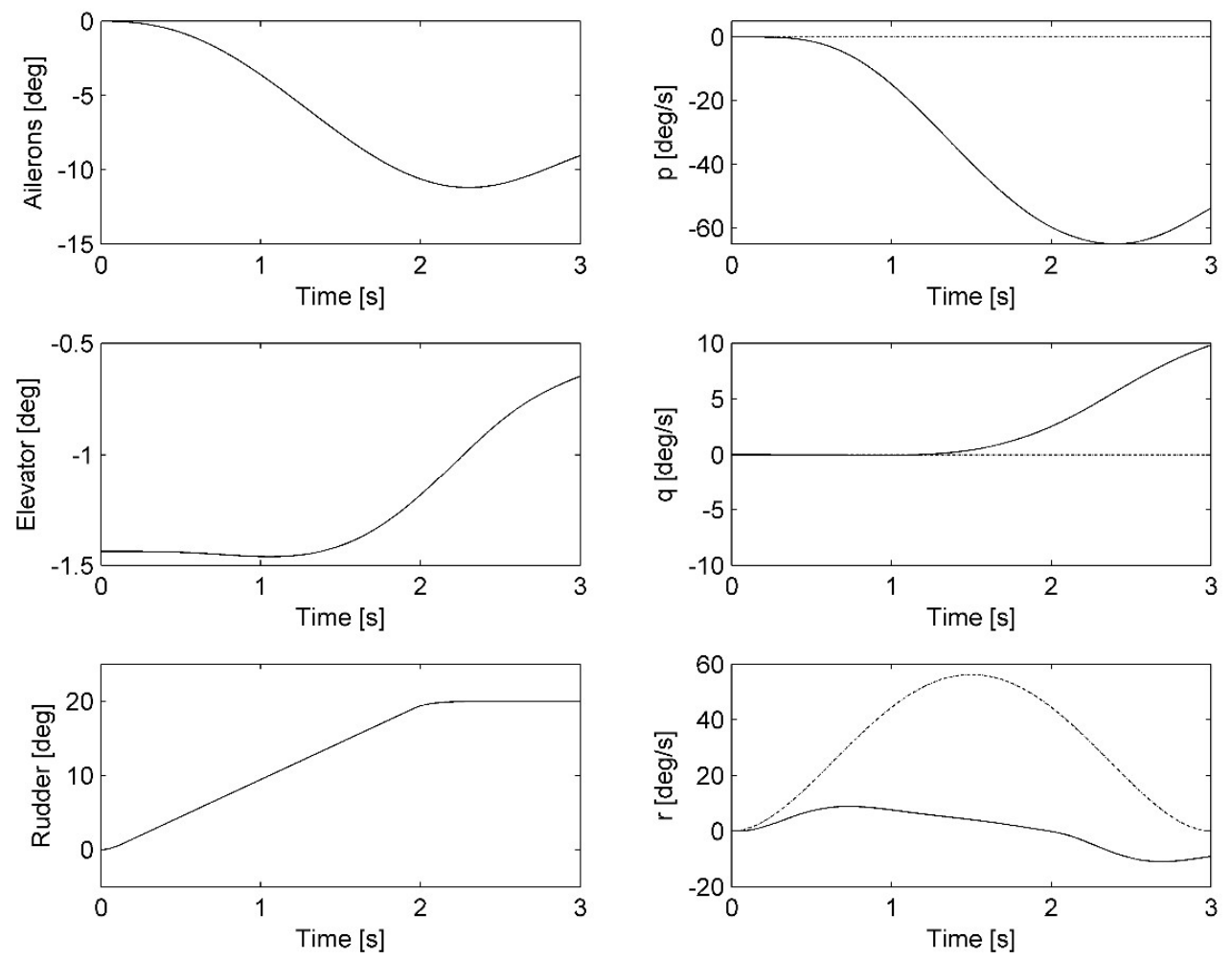

Figure 10. Control surface angular deflections and associated attitude rates found by the approximate differentiation method of inverse simulation for a case involving actuator amplitude limits of \pm 20 deg.and rate limits of actuator rate limits of $\pm 10 \mathrm{deg} / \mathrm{s}$ for a 
manoeuvre involving a maximum demanded yaw rate of $56.25 \mathrm{deg} / \mathrm{s}$. The desired angular velocities are shown by the dashed lines.

\section{Inverse simulation results with actuator limiting using the feedback approach}

Figure 11 shows results for a maximum demanded heading rate of $18.75 \mathrm{deg} / \mathrm{s}$ implemented within the feedback structure that was used previously. For this larger manoeuvre the rudder actuator reaches its amplitude limit. These are equivalent to the results shown in Figure 9 for the approximate differentiation method. 

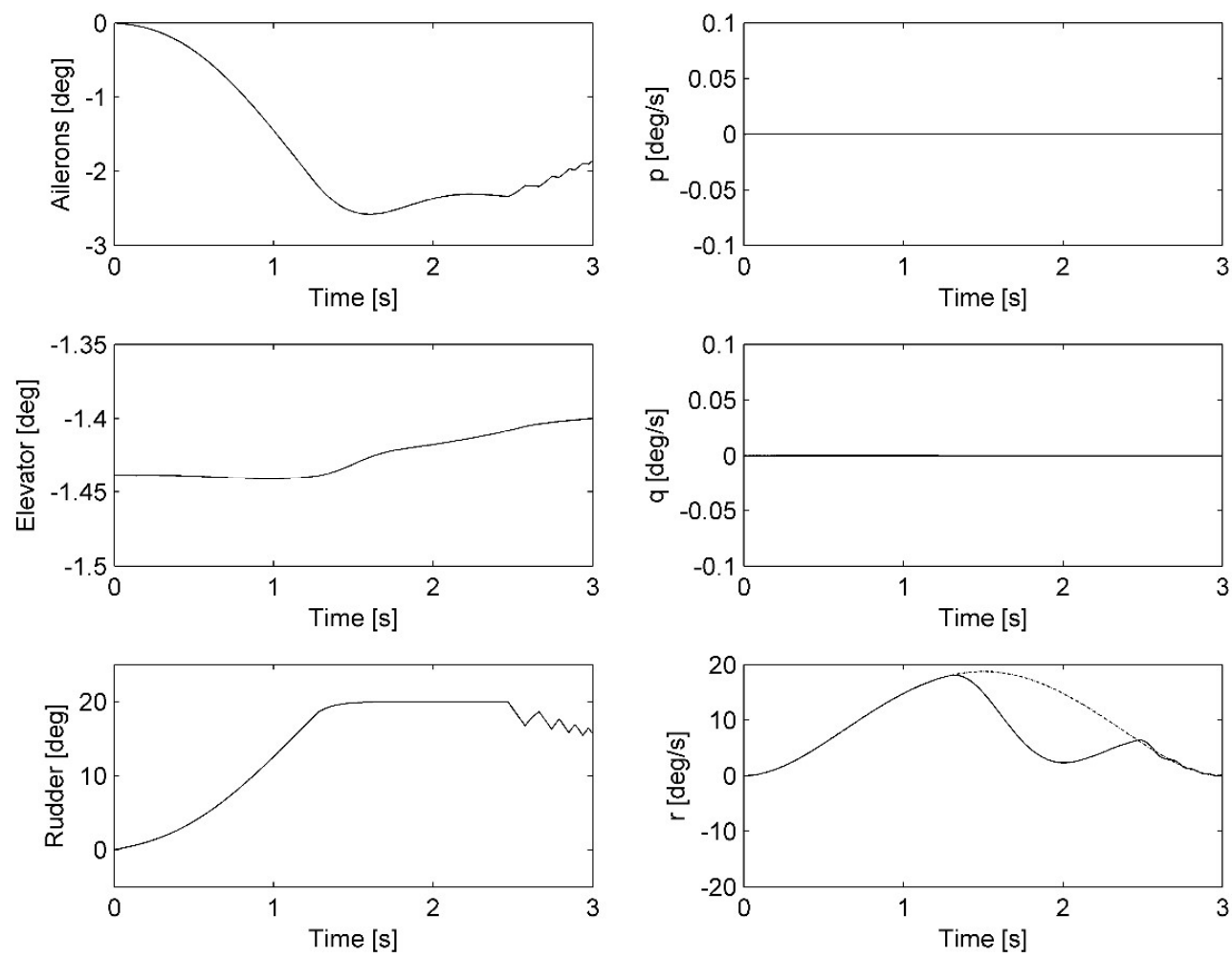

Figure 11. Control surface deflections and associated attitude rates found by the feedback method of inverse simulation for case of a manoeuvre involving a maximum yaw rate of $18.75 \mathrm{deg} / \mathrm{s}$, amplitude limits of $\pm 20 \mathrm{deg}$ and rate limits of $\pm 30 \mathrm{deg} / \mathrm{s}$. The desired angular velocities are shown by the dashed lines. 
Although the general form of the control surface time histories obtained by the feedback method are similar to those found by the two-stage approximate differentiation procedure, it is clear that, with the feedback method, the rudder comes out of saturation at about time $t=2.5 \mathrm{~s}$ whereas, with the approximate differentiation approach, the rudder remained at its amplitude limit. The magnitude of the control surface deflections for the ailerons and elevator are also slightly smaller for the feedback method. One point of detail is that the rudder deflection record shows an oscillation during the final stage when the rudder actuator comes out of saturation and this oscillatory response is rate limited. This oscillation has little effect on other variables of the model as it is of relatively high frequency compared with the dynamics of the forward model of the vehicle. For the variables $p$ and $q$, the angular rate values in Figure 11 are close to the required values of zero, in contrast to the values for the approximate differentiation method (shown in Figure 9). For the yaw rate, $r$, the form of the response in Figure 11 is similar to that in Figure 9 and the maximum is also similar and occurs at a similar time. 
Changing the rate limit from $\pm 30 \mathrm{deg} / \mathrm{s}$ to $\pm 10 \mathrm{deg} / \mathrm{s}$ and repeating the test shows very clearly the effect of the rate limit on the behaviour of the actuator (Figure 12). The time history of the rudder deflection is similar to that of Figure 10 for the approximate differentiation method for the same manoeuvre and the same rate limit. On the other hand, the results of Figure 12 show that, with the feedback approach, the pitch and roll rates are much closer to the desired values in than those found by the approximate differentiation method for the same conditions. However, it should be noted that the range of elevator and aileron deflections to achieve this is significantly different from those found by the approximate differentiation method and the smaller values of error associated with the pitch and roll rates is clearly due to the action of the high-gain feedback pathways. 

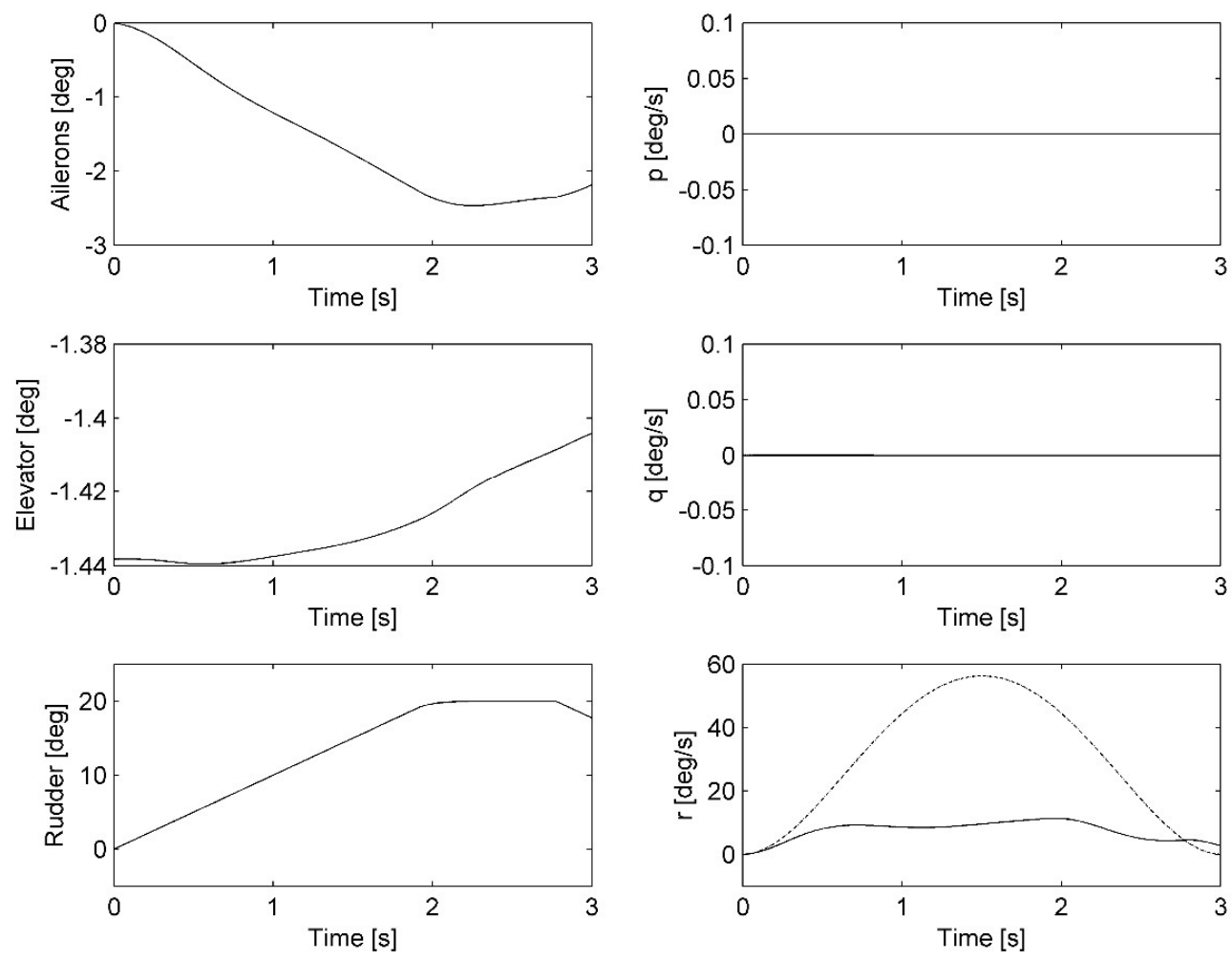

Figure 12: Control surface angular deflections and associated attitude rates found by the feedback method of inverse simulation for a case involving actuator amplitude limits of $\pm 20 \mathrm{deg}$ and rate limits of actuator rate limits of $\pm 10 \mathrm{deg} / \mathrm{s}$ for a manoeuvre involving a maximum demanded yaw rate of $56.25 \mathrm{deg} / \mathrm{s}$. The desired angular velocities are shown by the dashed lines. 


\section{Analysis of the feedback approach to inverse simulation}

Problems of instability or limit cycle oscillations have been reported in other applications of the high-gain feedback approach and more complex feedback structures based, for example, on eigenstructure assignment design methods have been applied in some cases to counter the problems of instability. ${ }^{21,23}$

Single-input single-output (SISO) models having non-minimum phase characteristics present particular difficulties in terms of instability of the inverse simulation model because right-half plane zeros in a forward model lead, inevitably, to right-half plane poles in the inverse. It might be considered surprising that problems of this kind have not been encountered in this case study, especially when it is noted that the transfer function descriptions, given in Appendix 2, include some cases with non-minimum phase characteristics. However, analysis based on the linearised model, and especially the twoinput two-output block diagram of Figure 15 (in Appendix 2), allows a better understanding to be gained of reasons for the success of the feedback approach in this multi-input multi-output (MIMO) case. 


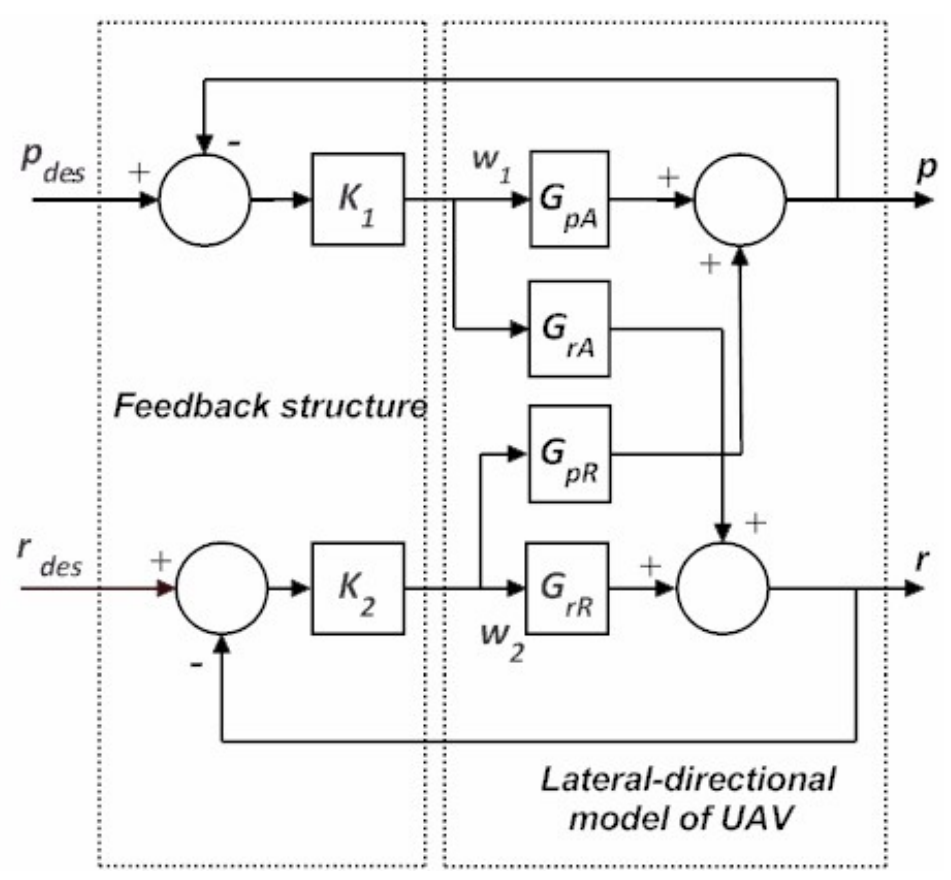

Figure 13. Block diagram of linearised lateral-directional model of UAV with feedback structure for inverse simulation.

Figure 13 shows the linearised model structure of Appendix 2 with high-gain feedback applied. There are two reference inputs representing the required time histories of the roll rate and yaw rate, which are the output variables. As for the SISO situation shown in Figure 2, the inverse solutions are obtained at the ouputs of the two controller blocks $K_{1}$ and $K_{2}$. Analysis of the model may be simplified using concepts developed for the 'individual channel' approach to the analysis and design of MIMO feedback systems. ${ }^{28}$ 
Through this the MIMO structure may be transformed into two SISO feedback loops with loop interactions preserved. Figure 14 shows block diagrams of the two single-loop representations and these, when taken together, are equivalent to the structure of Figure 13.

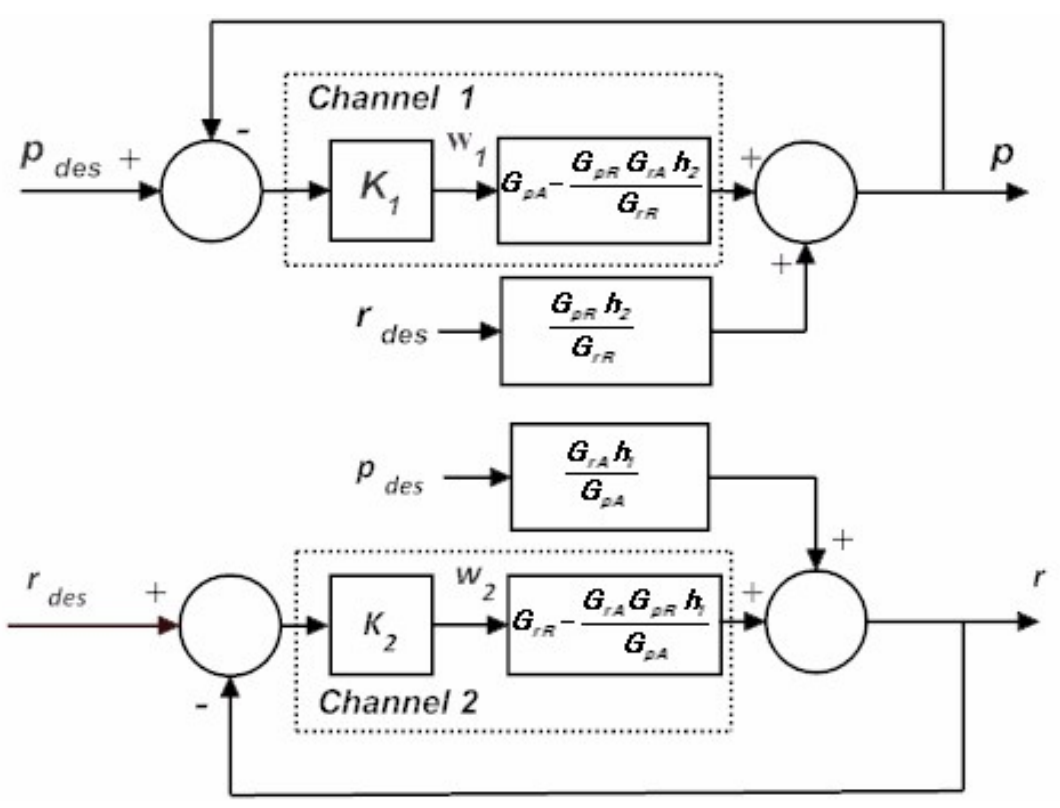

Figure 14. Structure of Channels 1 and 2 involving single-input single-output representations of the two-input system of Figure 13. Reference input $p_{\text {des }}$ is linked to output $p$ with an additional 'disturbance' pathway representing the effect of reference input $r_{\text {des. }}$ Similarly input $r_{\text {des }}$ is linked to output $r$ with a disturbance pathway representing 
the effect of reference input $p_{\text {des. }}$. For these outputs, this is equivalent to the structure of Figure 13.

The coupling pathways between Channel 1 and Channel 2 appear now as disturbance inputs and, because of the cross-coupling within this model, the transfer function relationships between the reference inputs $p_{d e s}$ and $r_{d e s}$ and the actuator inputs $w_{1}$ and $w_{2}$ (the inverse models) depend on all four of the system transfer functions.

The quantities $h_{1}$ and $h_{2}$ in these diagrams are defined as follows:

$$
\begin{gathered}
h_{1}=\frac{K_{1} G_{p A}}{1+K_{1} G_{p A}} \\
h_{2}=\frac{K_{2} G_{r R}}{1+K_{2} G_{r R}}
\end{gathered}
$$

If the reference input $r_{\text {des }}$ is zero, so that we are defining an output $p$ only, and if the gain factors $K_{1}$ and $K_{2}$ are chosen to have values tending to infinity, the signal $w_{1}$ can be shown to have the form:

$$
w_{1} \cong \frac{G_{r R}}{G_{p A} G_{r R}-G_{p r} G_{r A}} p_{d e s}
$$


Substituting the four transfer functions $G_{p A}, G_{r R}, G_{p r}$ and $G_{r A}$ of Appendix 2 allows the overall transfer function relating $w_{1}$ to $p_{\text {des }}$ to be evaluated. Following pole-zero cancellation using the MATLAB Control Systems Toolbox minreal function ${ }^{29}$ the resulting reducedorder transfer function is found to have zeros at $s=-11.3738, s=0.0341$ and $s=-1.7849$ $\pm \mathrm{j} 4.6213$. The poles lie at $s=0.0284$ and at $s=-1.7420 \pm j 4.6589$ and are therefore at the positions of the zeros of $G_{p A}$. It therefore follows that the zeros are at the positions of the poles of that transfer function. Similarly, if the reference input $p_{\text {des }}$ is zero so that we are defining an output $r$ only, the signal $w_{2}$ has the form:

$$
w_{2} \cong \frac{G_{p A}}{G_{p A} G_{r R}-G_{p r} G_{r A}} r_{d e s}
$$

In this case the transfer function from $r_{\text {des }}$ to $w_{2}$ is found to have poles at $s=-11.3403$ and at $s=-0.2908 \pm j 0.6882$. The zeros of the transfer function are at $s=-11.3738, s=$ 0.0341 and at $\mathrm{s}=-1.7849 \pm j 4.6213$. The poles and zeros of this transfer function are thus the same as the zeros and poles, respectively, of the transfer function $G_{r R}$.

It can be seen that the inverse models have the form that would be expected from the SISO case. They have poles in the right-half of the $s$-plane, corresponding to the positions of non-minimum phase zeros, but these are located close to the positions of the poles of the forward model (which become zeros in the inverse) and their effects are partially 
cancelled. Over a period of 30 seconds the unstable modes, in both the forward and inverse models, do produce an apparent drift but, for the much shorter periods over which responses are considered in manoeuvring flight or in handling qualities investigations, this is not significant.

\section{Discussion and conclusions}

This study, which has involved application of two continuous system approaches to inverse simulation of a nonlinear MIMO model of a UAV, has highlighted several issues. These relate both to the methods used and to the application, especially in terms of issues that can arise in dealing with hard nonlinearities, such as actuator saturation and rate limiting.

The results obtained using the two methods are almost identical for cases in which the model does not include actuator nonlinearities. However, the approximate differentiation method involves more preparatory work since the given model has to be altered in structure from the state-space form used for forward simulation. Any changes introduced at a later stage could result in further algebraic manipulation to include those changes in the inverse simulation. However, the feedback approach simply involves the application of feedback to the forward simulation and thus avoids the need to change the inverse simulation 
structure when alterations are made. Unfortunately, the feedback can lead to further problems of instability and limit cycling ${ }^{21-23}$, and to other limitations, as discussed below.

Actuator nonlinearities cannot be included within the inverse simulation using the approximate differentiation method and a two-stage approach has to be adopted. This is satisfactory for detecting the onset of actuator limiting for a given manoeuvre and could therefore be useful within the design process for investigating of design limits and for path planning.

For the feedback approach few difficulties arise, even if actuator nonlinearities are included. Feedback can be applied around the complete system model and it might be assumed that the effect of actuator nonlinearities on the performance could be observed directly. However, it is clear that the feedback approach to inverse simulation is useful only when the system model has not reached any hard limit. From the moment when saturation or rate limiting occurs until the time when the actuator sub-model comes out of the limited condition again, the feedback loop is inactive. Since the basis of this feedback approach to inverse simulation depends upon error-actuated feedback loops this suggests that there are limits to the applicability of this methodology. However, for most practical applications 
involving amplitude and rate limited actuators, interest is focussed on the detection of conditions where limiting occurs and avoiding the occurrence of limiting rather than on finding the complete inverse response once the system has limited. The feedback approach is well suited to doing this directly. However, a two-stage approach, similar to that used with the approximate differentiation method, could also be used in situations where the feedback approach with actuator nonlinearities proves difficult to apply.

It can be concluded that the continuous simulation methods considered provide useful alternatives to conventional iterative methods for inverse simulation based on discrete models. In particular, the continuous approach avoids issues associated with choice of sampling rates that arise with inverse simulation methods based on discretised models. Both the approaches considered have been shown to provide useful information about the onset of actuator limiting for specific demanded manoeuvres. Although the emphasis in this paper is on a nonlinear model, the use of linear methods of analysis has also provided useful insight. The ICAD methodology has provided insight about inverse solutions that could not have been gained so readily using conventional simulation methods. 


\section{References}

1. Thomson DG and Bradley R. The principles and practical application of helicopter inverse simulation. Simulation Practice and Theory. 1998; 6 (1): 47-70.

2. Thomson D and Bradley R. Inverse simulation as a tool for flight dynamics researchPrinciples and applications. Prog. in Aerospace Sciences. 2006; 42: 174-210.

3. Hess RA, Gao C and Wang SH. A generalized technique for inverse simulation applied to aircraft maneuvers. AIAA J. Guidance Control and Dynamics. 1991; 14 (5): 920-926.

4. Rutherford S and Thomson DG. Improved methodology for inverse simulation. Aeronautical J. 1996; 100 (993): 79-86.

5. Lu L, Murray-Smith DJ and Thomson DG. Issues of numerical accuracy and stability in inverse simulation, Simulation Modelling Practice and Theory. 2008;16: 1350-1364.

6. Thomson DG and Bradley R. An analytical method for quantifying helicopter agility. In: $12^{\text {th }}$ European Rotorcraft Forum, Garmisch-Partenkirchen, Federal Republic of Germany, September 1986. Paper 45.

7. Thomson DG and Bradley R. Recent developments in the calculation of inverse solutions of the helicopter equations of motion. In: UK Simulation Council Conference, 
University College of North Wales, 9-11 September 1987, Ghent, Belgium: UKSC,1987, pp 227-234.

8. Kato $\mathrm{O}$ and Suguira I. An interpretation of airplane motion and control as inverse problems, AIAA J. Guidance, Control and Dynamics, 9 (2) (1986), 198-204.

9. Celi R. Optimisation based inverse simulation of a helicopter slalom maneuver, AIAA J. Guidance Control and Dynamics. 2000; 23 (2): 289-297.

10. Lee S and Kim Y. Time domain finite element method for inverse problem of aircraft maneuvers. AIAA J. Guidance, Control and Dynamics. 1997; 20 (1): 97-103.

11. Murray-Smith DJ. Modelling and Simulation of Integrated Systems in Engineering. Cambridge, UK: Woodhead, Cambridge, 2012, Chapter 4.

12. Blajer W and Kołodziejczky K. Improved DAE formulation for inverse dynamics simulation of cranes. Multibody System Dynamics. 2011; 25 (2): 131-143.

13. Thümme M, Looye G, Kurze M, Otter M and Bals J. Nonlinear inverse models for control. In: Proceedings of the $4^{\text {th }}$ International Modelica Conference, (ed. G. Schmitz), Hamburg, Germany, 7-8 March 2005. Linköping, Sweden; Modelica Organisation: 2005, pp 267-279. 
14. Murray-Smith DJ. Looking at problems the other way round: engineering applications of inverse simulation based on continuous system simulation methods. In; $8^{\text {th }}$ EUROSIM Congress, Cardiff, Wales, UK, September 10-12 2013. Invited Keynote Lecture No. 4, http://eurosim2013.info/. (2013, accessed 21 March 2014).

15. Hamel PG. Aerospace vehicle modelling requirements for high bandwidth flight control. In, Cook MV and Rycroft MJ (eds.), Aerospace Vehicle Dynamics and Control, Oxford, UK; Clarendon Press: 1994, pp. 1-31

16 . von Grünhagen W, Bouwer G, Pausder HJ, Henshel F and Kaletka J. A high bandwidth control system for the helicopter in-flight simulator ATTHeS - modelling, performance and applications. In, Tischler MB (ed.), Advances in Aircraft Flight Control. London, UK; Taylor and Francis: 1996, pp. 73-101.

17. Buchholz JJ and von Grünhagen W. Inversion Impossible?. Bremen, Germany: University of Applied Sciences, Technical Report, 2004.

18. Tagawa $\mathrm{Y}$ and Fukui K. Inverse dynamics calculation of nonlinear model using low sensitivity compensator. In: Proc. of Dynamics and Design Conf., 1994, pp. 185-188.

19. Venture G, Kojima T and Tagawa Y. Fast motion control of robotic systems using inverse dynamics compensation via 'simulation of feedback control systems' (IDCS). In: 
Proc. $1^{\text {st }}$ Joint Conf. on Multibody System Dynamics, May 25-27 2010, Lapeenranta, Finland.

20. Tagawa Y, Tu JY and Stoten DP. Inverse dynamics compensation via 'simulation of feedback control systems', Proc. Institution of Mechanical Engineers, Part I: J. Systems and Control Eng., 225 (2012), pp. 137-153.

21. Murray-Smith DJ. Feedback methods for inverse simulation of dynamic models for engineering system applications, Mathematical and Computer Modelling of Dynamical Systems. 2011; 17 (5): 515-541.

22. Murray-Smith DJ. The application of parameter sensitivity analysis methods to inverse simulation models, Mathematical and Computer Modelling of Dynamical Systems. 2013: 19 (1), 67-90.

23. Murray-Smith DJ. Inverse simulation and analysis of underwater vehicle dynamics using feedback principle, Mathematical and Computer Modelling of Dynamical Systems, 2014: $20(1), 45-65$.

24. Fielding C and Flux PK. Nonlinearities in flight control systems. Aeronautical J., 2003: 107, 673-696. 
25. Ducard GGJ, Fault-tolerant flight control and guidance systems: practical methods for small unmanned aerial vehicles. London, UK: Springer-Verlag, 2009.

26. Winit R. Sensor fault-tolerant control system for unmanned aerial vehicle, University of Glasgow, 2010. MSc Thesis.

27. Lone MM and Cooke AK. Effects of nonlinear flight control system elements on aircraft manual control, Cranfield University, 2011. College of Aeronautics Report No. CU/COA-2011/01.

https://dspace.lib.cranfield.ac.uk/bitstream/1826/6161/1/COA_2011_01-2011.pdf (accessed 28 June 2014).

28. O’Reilly, J and Leithead WE. Multivariable control by ‘individual channel design’, Int. J. Control, 1991, 54 (1): 1-46.

29. MathWorks, Documentation, Control Systems Toolbox ${ }^{\mathrm{TM}}$, http://www.mathworks.co.uk/help/toolbox/control/ (accessed 21 March 2014). 
The fixed wing UAV model used in this study is described by a nonlinear six-degreeof-freedom model, involving an Earth-fixed navigation frame and a body frame, as shown in Figure $3 .^{24,25}$ The airflow is represented by an airspeed vector, magnitude $V_{\mathrm{T}}$ and direction relative to the airframe defined by angle of attack $\alpha$ and sideslip angle $\beta$. A coordinate frame is defined (the wind axes) such that the $x$ axis in this frame points along the airspeed vector.

Symbols used for variables and parameters of this model are consistent with those used in aeronautical engineering. ${ }^{24}$ Parameter values are provided in Table 3.

The equations form a conventional nonlinear state-space type of description:

$$
\begin{aligned}
& \dot{u}=-g \sin \theta+r v-q w+\frac{\left(F_{T}+X\right)}{\bar{m}} \\
& \dot{v}=g \sin \phi \cos \theta+p w-r u+\frac{Y}{\bar{m}} \\
& \dot{w}=g \cos \phi \cos \theta+q u-p v+\frac{z}{\bar{m}} \\
& \dot{p}=P_{p q} p q+P_{q r} q r+P_{N} N+P_{L} L \\
& \dot{q}=Q_{p p} p^{2}+Q_{p r} p r+Q_{r r} r^{2}+Q_{M} M \\
& \dot{r}=R_{p q} p q+R_{q r} q r+R_{N} N+R_{L} L
\end{aligned}
$$




$$
\begin{aligned}
& \dot{\phi}=p+q \tan \theta \sin \phi+r \cos \phi \tan \theta \\
& \dot{\theta}=q \cos \phi-r \sin \phi \\
& \dot{\psi}=g \sin \phi \sec \theta+r \cos \phi \sec \theta \\
& \dot{x}_{n}=u \cos \theta \cos \psi+v \cos \theta \sin \psi-w \sin \theta \\
& \dot{y}_{n}=u(\sin \phi \sin \theta \cos \psi-\cos \phi \sin \psi)+v(\sin \phi \sin \theta \sin \psi+\cos \phi \cos \psi) \\
& \quad \quad-w \sin \phi \cos \theta \\
& \dot{z}_{n}=u(\cos \phi \sin \theta \cos \psi+\sin \phi \sin \psi)+v(\cos \phi \sin \theta \sin \psi-\sin \phi \cos \psi) \\
& \quad+w \cos \phi \cos \theta \\
& \dot{n}=-\frac{1}{\tau_{n}} n+\frac{1}{\tau_{n}} n_{c} \\
& \dot{\alpha}=\frac{1}{\cos \beta}\left(q_{w}+\frac{1}{\bar{m} V_{T}}\left[Z_{w}-F_{T} \sin \alpha+m g_{z}^{w}\right]\right) \\
& \dot{\beta}=-r_{w}+\frac{1}{m V_{T}}\left[Y_{w}-F_{T} \cos \alpha \sin \beta+m g_{y}^{w}\right] \\
& \dot{V}_{T}=\frac{1}{\bar{m}}\left[X_{w}+F_{T} \cos \alpha \cos \beta+m g_{x}^{w}\right]
\end{aligned}
$$

The state variables are referred to the body-fixed frame of reference (unless stated otherwise) and are as given in Table 2.

In equation (22) the variable $F_{T}$ represents the thrust force which is given by:

$$
F_{T}=\rho D^{4}\left(C_{F T 1}+J C_{F T}+J^{2} C_{F T}\right) n^{2}
$$


where $J=\frac{V_{T}}{\pi D n}$ is termed the thrust advance ratio and the other quantities are as given in Table 3.

Variables $X, Y$ and $Z$ in equations (22) - (24) are aerodynamic forces. In the wind frame these are:

$$
\left[\begin{array}{c}
X_{w} \\
Y_{w} \\
Z_{w}
\end{array}\right]=\bar{q} S\left[\begin{array}{c}
C_{X 1}+C_{X \alpha} \alpha+C_{X \alpha^{2}} \alpha^{2}+C_{X \beta^{2}} \beta^{2} \\
C_{Y 1} \beta \\
C_{Z 1}+C_{Z \alpha} \alpha
\end{array}\right]
$$

and can be translated to the body-fixed frame. ${ }^{24}$ Other quantities in equation (39) are given in Table 3. 
Table 3. Parameters of the UAV model. ${ }^{24}$

\begin{tabular}{|c|c|c|c|c|c|}
\hline Parameter & Description & Value & Parameter & Description & Value \\
\hline $\bar{m}$ & Mass (kg) & 28 & $\rho$ & $\begin{array}{l}\text { Air density } \quad(\mathrm{kg} \\
\left.\mathrm{m}^{-3}\right)\end{array}$ & 1.166 \\
\hline$g$ & $\begin{array}{l}\text { Gravitational } \\
\text { constant }\left(\mathrm{m} \mathrm{s}^{-2}\right)\end{array}$ & 9.81 & $D$ & $\begin{array}{l}\text { Propeller diameter } \\
\text { (m) }\end{array}$ & 0.79 \\
\hline$I_{\mathrm{xx}}$ & $\begin{array}{l}\text { Roll axis moment } \\
\text { of inertia }\left(\mathrm{kg} \mathrm{m}^{2}\right)\end{array}$ & 2.56 & $\tau_{\mathrm{n}}$ & $\begin{array}{l}\text { Engine time constant } \\
\text { (s) }\end{array}$ & 0.4 \\
\hline$I_{\mathrm{yy}}$ & $\begin{array}{l}\text { Pitch axis moment } \\
\text { of inertia }\left(\mathrm{kg} \mathrm{m}^{2}\right)\end{array}$ & 10.9 & $\begin{array}{l}C_{\mathrm{FT} 1}, \\
C_{\mathrm{FT} 2}, \\
C_{\mathrm{FT} 3}\end{array}$ & $\begin{array}{l}\text { Thrust force } \\
\text { derivatives }\end{array}$ & $\begin{array}{c}0.0842 \\
0.000 \\
-0.928\end{array}$ \\
\hline$I_{\mathrm{zz}}$ & $\begin{array}{l}\text { Yaw axis moment } \\
\text { of inertia }\left(\mathrm{kg} \mathrm{m}^{2}\right)\end{array}$ & 11.3 & $\begin{array}{c}C_{\mathrm{La}}, \\
C_{\mathrm{L} \beta}, \\
C_{\mathrm{L}} \tilde{p}, \\
C \tilde{r}\end{array}$ & Roll derivatives & $\begin{array}{c}0.0679, \\
-0.0130, \\
-0.1920, \\
0.0361\end{array}$ \\
\hline$I_{\mathrm{xy}}, I_{\mathrm{yx}}, I_{\mathrm{zy}}$ & $\begin{array}{l}\text { Products of inertia } \\
\left(\mathrm{kg} \mathrm{m}^{-2}\right)\end{array}$ & 0.0 & $\begin{array}{l}C_{\mathrm{M} 1}, \\
C_{\mathrm{Me}}, \\
C_{\mathrm{M}} \widetilde{q} \\
C_{\mathrm{M} \alpha}\end{array}$ & Pitch derivatives & $\begin{array}{c}0.0208 \\
0.5450 \\
-9.8300 \\
-0.0903\end{array}$ \\
\hline$I_{\mathrm{xz}}, I_{\mathrm{zx}}$ & $\begin{array}{l}\text { Products of inertia } \\
\left(\mathrm{kg} \mathrm{m}^{-2}\right)\end{array}$ & 0.5 & $\begin{array}{l}C_{\mathrm{N} \delta \mathrm{r}}, \\
C_{\mathrm{N}} \tilde{r} \\
C_{\mathrm{N} \beta}\end{array}$ & Yaw derivatives & $\begin{array}{c}0.0534 \\
-0.2140 \\
0.0867\end{array}$ \\
\hline$S$ & $\begin{array}{l}\text { Wing surface area } \\
\left(\mathrm{m}^{2}\right)\end{array}$ & 1.8 & $\begin{array}{l}C_{\mathrm{Zl}}, \\
C_{\mathrm{Z} \alpha}\end{array}$ & Lift derivatives & $\begin{array}{l}0.0129 \\
-3.2500\end{array}$ \\
\hline$b$ & Wing span (m) & 3.1 & $\begin{array}{l}C_{\mathrm{X} 1}, \\
C_{\mathrm{X} \alpha}, \\
C_{\mathrm{X}{ }^{2}}{ }^{2} \\
C_{\mathrm{X} \beta}{ }^{2}\end{array}$ & Drag derivatives & $\begin{array}{c}-0.0212, \\
-0.0266, \\
-1.550 \\
-0.4010\end{array}$ \\
\hline $\bar{c}$ & $\begin{array}{l}\text { Mean aerodynamic } \\
\text { chord }(\mathrm{m})\end{array}$ & 0.58 & $C_{\mathrm{Y} 1}$ & Side slip derivative & -0.3790 \\
\hline
\end{tabular}


In equations (25) - (27) the torques $L, M$ and $N$ developed by the control surfaces are expressed in the body-fixed frame as:

$$
\left[\begin{array}{c}
L \\
M \\
N
\end{array}\right]=\bar{q} S\left[\begin{array}{c}
b\left(C_{L \beta} \beta+C_{L \tilde{p}} \tilde{p}+C_{L \tilde{r}} \bar{r}+C_{L a} \delta_{a}\right) \\
\bar{c}\left(C_{M 1}+C_{M \bar{q}} \tilde{q}+C_{M \alpha} \alpha+C_{M e} \delta_{e}\right) \\
b\left(C_{N \beta} \beta+C_{N \tilde{r}} \tilde{r}+C_{N r} \delta_{r}\right)
\end{array}\right]
$$

where $\delta_{a}, \delta_{e}$ and $\delta_{r}$ are control surface angular deflections and other quantities are as given in Table 3. The variable $\bar{q}$ is defined as $\bar{q}=\rho V_{T}$ where $\rho$ is the air density. The quantities $\tilde{p}, \tilde{q}$ and $\tilde{r}$ are:

$$
\begin{aligned}
& \tilde{p}=\frac{b p}{2 V_{T}} \\
& \tilde{q}=\frac{\bar{c} q}{2 V_{T}} \\
& \bar{r}=\frac{b r}{2 V_{T}}
\end{aligned}
$$

Other quantities in equation (40) are defined in Table 3.

In equations (25) - (27) the quantities $P_{p q}, P_{q r}, Q_{p p}, Q_{p r}, Q_{r r}, R_{p q}, R_{q r}, P_{L}, P_{N}$, $Q_{M}, Q_{r r}, R_{L}$ and $R_{N}$ are:

$$
P_{p q}=\frac{I_{x z}\left(I_{x x}-I_{y y}+I_{z z}\right)}{I_{x x} I_{z z}-I_{x z}^{2}}
$$




$$
\begin{aligned}
P_{q r} & =\frac{\left(I_{y y}-I_{z z}\right) I_{z z}-I_{x z}^{2}}{I_{x x} I_{z z}-I_{x z}^{2}} \\
Q_{p p} & =-\frac{I_{x z}}{I_{y y}} \\
Q_{p r} & =\frac{I_{z z}-I_{y y}}{I_{y y}} \\
R_{p q} & =\frac{\left(I_{x x}-I_{y y}\right) I_{x x}+I_{x z}^{2}}{I_{x x} I_{z z}-I_{x z}^{2}} \\
R_{q r} & =\frac{I_{x z}\left(I_{y y}-I_{z z}-I_{x x}\right)}{I_{x x} I_{z z}-I_{x z}^{2}} \\
P_{L} & =\frac{I_{z z}}{I_{x x} I_{z z}-I_{x z}^{2}} \\
P_{N} & =\frac{I_{x z}}{I_{x x} I_{z z}-I_{x z}^{2}} \\
R_{N} & =\frac{I_{x x}}{I_{x x} I_{z z}-I_{x z}^{2}} \\
Q_{M} & =\frac{1}{I_{y y}} \\
Q_{r r} I_{z z}-I_{x z}^{2} & \frac{I_{x z}}{I_{y y}} \\
R_{x y} &
\end{aligned}
$$


In equations (35) - (37) the quantities $g_{x}^{w}, g_{y}^{w}$ and $g_{z}^{w}$ are the $x, y$ and $z$ components of the gravitational acceleration, all referred to the wind frame.

\section{Appendix 2. Linearised model of the UAV.}

A linearised model is available for an operating condition involving straight and level flight with a forward velocity of $30 \mathrm{~m} / \mathrm{s}^{26}$ This is close to the initial conditions used in the simulation studies. Coupling between the longitudinal and lateral dynamics is not strong and the longitudinal and lateral sub-models can be considered separately.

The linearised model for longitudinal variables has the form $\dot{\boldsymbol{x}}=\boldsymbol{A} \boldsymbol{x}+\boldsymbol{B} \boldsymbol{u}$ where the vector of state variables is $\boldsymbol{x}=\left[\begin{array}{lll}q & V_{T} \alpha & \theta\end{array}\right]^{T}$ and the input vector is $u=\delta_{e}$ where $\delta_{e}$ represents the elevator deflection perturbation. For the chosen operating point the system matrix $\boldsymbol{A}_{\text {long }}$ and input matrix $\boldsymbol{B}_{\text {long }}$ are:

$$
\begin{aligned}
\boldsymbol{A}_{\text {long }} & =\left[\begin{array}{cccc}
-4.7796 & 0 & -4.5420 & 0 \\
0 & -0.0830 & -0.8660 & -9.81 \\
1 & 0.0215 & -3.6573 & 0 \\
1 & 0 & 0 & 0
\end{array}\right] \\
\boldsymbol{B}_{\text {long }} & =\left[\begin{array}{c}
27.4128 \\
0 \\
0 \\
0
\end{array}\right]
\end{aligned}
$$


The transfer function relating the pitch rate perturbation $q$ to the elevator perturbation $\delta_{e}$ is:

$$
\begin{aligned}
G_{q E}= & \frac{27.41 s^{3}+102.5 s^{2}+7.811 s+5.135 \times 10^{-15}}{s^{4}+8.52 s^{3}+22.7 s^{2}+1.789 s+0.958} \\
& \cong \frac{s\left(27.41 s^{2}+102.5 s+7.811\right)}{s^{4}+8.52 s^{3}+22.7 s^{2}+1.789 s+.958}
\end{aligned}
$$

Poles are at $s=-4.2289 \pm \mathrm{j} 2.0621 \mathrm{rad} / \mathrm{s}$ (the short period mode) and at $s=-0.0310 \pm \mathrm{j}$ $0.2057 \mathrm{rad} / \mathrm{s}$ (the phugoid mode). Zeros lie at $s=0.0000,-0.0778$ and $-3.6625 \mathrm{rad} / \mathrm{s}$.

The lateral state variables are $\boldsymbol{x}=\left[\begin{array}{llll}p & r & \beta & \phi\end{array}\right]^{T}$ with input $\boldsymbol{u}=\left[\begin{array}{ll}\delta_{a} & \delta_{r}\end{array}\right]^{T}$. For the chosen operating point the system matrix $\boldsymbol{A}_{\text {lat }}$ and input matrix $\boldsymbol{B}_{\text {lat }}$ are:

$$
\begin{aligned}
\boldsymbol{A}_{\text {lat }} & =\left[\begin{array}{cccc}
-11.4540 & 2.7185 & -19.4390 & 0 \\
0.5068 & -2.9875 & 23.3434 & 0 \\
0.0922 & -0.9957 & -0.4680 & 0.3256 \\
1 & 0.0926 & 0 & 0
\end{array}\right] \\
\boldsymbol{B}_{\text {lat }} & =\left[\begin{array}{cc}
78.4002 & -2.7282 \\
-3.4690 & 13.9685 \\
0 & 0 \\
0 & 0
\end{array}\right]
\end{aligned}
$$

The model defined by these equations is represented by the two-input two-output block diagram of Figure 15 where the inputs are the aileron perturbation $\delta_{a}$ and the rudder perturbation $\delta_{r}$. Output variables are the roll rate perturbation, $p$, and the yaw rate $r$. 


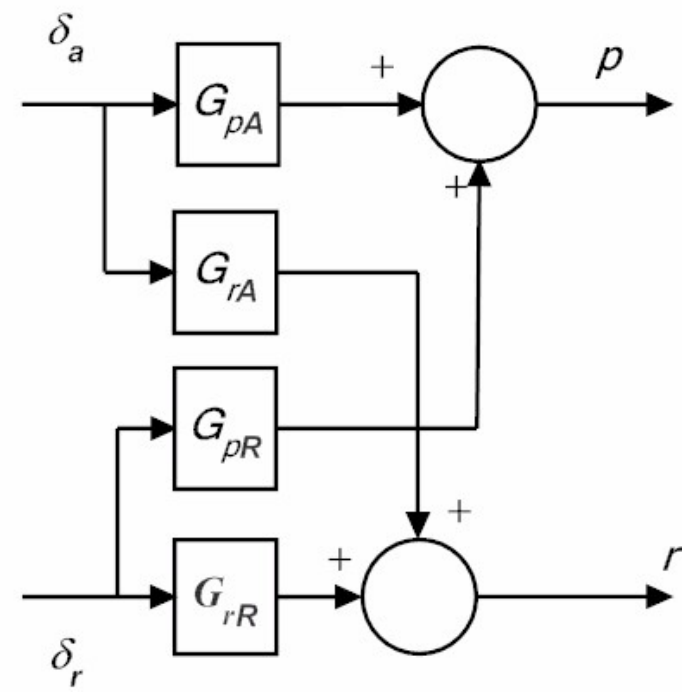

Figure 15. Block diagram of the two-input two-output linearised model for lateral dynamics of the UAV.

The transfer function relating the roll rate perturbation $p$ in response to the aileron input perturbation $\delta_{a}$ has the form:

$G_{p A}=\frac{78.4 s^{3}+270.9+1932 s-55.18}{s^{4}+1.91 s^{3}+64.64 s^{2}+276.7 s-9.517}$

Poles of this transfer function are at $s=-1.7849 \pm \mathrm{j} 4.6213 \mathrm{rad} / \mathrm{s}$ (the dutch roll mode), at $s=0.0341 \mathrm{rad} / \mathrm{s}$ (the spiral mode) and at $s=-11.3738 \mathrm{rad} / \mathrm{s}$ (the roll subsidence mode). The zeros are at $s=0.0284 \mathrm{rad} / \mathrm{s}$ and at $s=-1.742 \pm \mathrm{j} 4.6589 \mathrm{rad} / \mathrm{s}$. This transfer function 
is thus non-minimum phase. The unstable pole associated with the spiral mode has a very low frequency compared with the other poles and has a zero close to it, thus tending to reduce the influence of this mode.

Similarly, the transfer function relating the yaw rate perturbation $r$ to the rudder input perturbation $\delta_{r}$ has the form:

$$
G_{r R}=\frac{13.97 s^{3}+166.5 s^{2}+99.91 s+8.42}{s^{4}+14.91 s^{3}+64.64 s^{2}+276.7 s-9.517}
$$

The poles of this transfer function are the same as those for the transfer function $G_{p A}$. The zeros are at $s=-11.4667 \mathrm{rad} / \mathrm{s}$ and at $s=-0.2908 \pm \mathrm{j} 4.6213 \mathrm{rad} / \mathrm{s}$. This transfer function is thus minimum phase but is unstable due to the presence of the pole at $s=$ $0.0341 \mathrm{rad} / \mathrm{s}$.

The transfer function relating perturbations of the roll rate $p$ in response to perturbations of the rudder $\delta_{r}$ is

$$
G_{p R}=\frac{-2.7285 s^{3}-9.427 s^{2}-67.23 s+1.92}{s^{4}+14.91 s^{3}+64.64 s^{2}+27.7 s-9.517}
$$


and the transfer function relating perturbations of the yaw rate $r$ in response to perturbations of the ailerons $\delta_{a}$ is

$$
G_{r A}=\frac{-3.496 s^{3}-41.68 s^{2}-25.01 s-\quad .13}{s^{4}+1.91 s^{3}+64.64 s^{2}+27.7 s-9.517}
$$

Note that the poles of $G_{r A}$ and $G_{p R}$ are the same as those for the transfer function $G_{p A}$. 